\title{
Interpolation by $G^{2}$ Quintic Pythagorean-Hodograph Curves
}

\author{
Gašper Jaklič ${ }^{1}$, Jernej Kozak ${ }^{2}$, Marjeta Krajnc ${ }^{2}$, Vito Vitrih ${ }^{3, *}$ and \\ Emil Žagar ${ }^{2}$ \\ ${ }^{1}$ FMF, University of Ljubljana, and IAM, University of Primorska, Jadranska \\ 19, 1000 Ljubljana, Slovenia. \\ 2 FMF and IMFM, University of Ljubljana, Jadranska 19, 1000 Ljubljana, \\ Slovenia. \\ ${ }^{3}$ FAMNIT and IAM, University of Primorska, Muzejski trg 2, 6000 Koper, \\ Slovenia.
}

Received 10 April 2013; Accepted (in revised version) 29 January 2014

Available online 12 August 2014

\begin{abstract}
In this paper, the $G^{2}$ interpolation by Pythagorean-hodograph (PH) quintic curves in $\mathbb{R}^{d}, d \geq 2$, is considered. The obtained results turn out as a useful tool in practical applications. Independently of the dimension $d$, they supply a $G^{2}$ quintic PH spline that locally interpolates two points, two tangent directions and two curvature vectors at these points. The interpolation problem considered is reduced to a system of two polynomial equations involving only tangent lengths of the interpolating curve as unknowns. Although several solutions might exist, the way to obtain the most promising one is suggested based on a thorough asymptotic analysis of the smooth data case. The numerical algorithm traces this solution from a particular set of data to the general case by a homotopy continuation method. Numerical examples confirm the efficiency of the proposed method.
\end{abstract}

AMS subject classifications: 65D05, 65D17

Key words: Pythagorean-hodograph curve, Hermite interpolation, geometric continuity, nonlinear analysis, homotopy.

\section{Introduction}

Polynomial Pythagorean-hodograph (PH) curves form a special subclass of parametric curves and have several important properties, such as a (piecewise) polynomial

\footnotetext{
${ }^{*}$ Corresponding author. Email addresses: gasper.jaklic@fmf.uni-lj.si (G. Jaklič), jernej.kozak@fmf.uni-lj.si (J. Kozak), marjetka.krajnc@fmf.uni-lj.si (M. Krajnc), vito.vitrih@upr.si (V. Vitrih), emil.zagar@fmf.uni-lj.si (E. Žagar)
} 
arc length, planar $\mathrm{PH}$ curves have rational offsets and spatial $\mathrm{PH}$ curves possess rational adapted frames. By definition, a parametric polynomial curve $\boldsymbol{p}:[0,1] \rightarrow \mathbb{R}^{d}$ of degree $\leq n$ is a PH curve if the Euclidean norm of its hodograph

$$
\left\|p^{\prime}\right\|=\sqrt{\boldsymbol{p}^{T} \boldsymbol{p}^{\prime}}
$$

is a piecewise polynomial of degree $\leq n-1$. They were first systematically studied in [10]. Since then, a lot of research work was put into studying them in detail.

A polynomial $\mathrm{PH}$ curve is usually obtained by the integration of the hodograph, constructed in a particular way from a so called preimage, which is a complex polynomial for a planar PH curve and a quaternion polynomial for a spatial $\mathrm{PH}$ curve (see e.g. $[7,8])$. A generalization to higher dimensional curves involves tools from Clifford algebras $[5,22]$. These constructions have many nice properties, specially from the computational point of view, but might not be so convenient when dealing with highly nonlinear geometric interpolation problems where the curve is completely determined only from geometric quantities like points, tangent directions and curvatures. Namely, to determine a geometric interpolant one needs to compute also the unknown parameters at which the points are interpolated, unknown tangent lengths, etc., and it is very desirable that these type of unknowns (geometric parameters) are separated from the unknown coefficients of the curve. When the curve is constructed from a complex or a quaternion polynomial, a separation of unknowns is difficult to achieve unless one increases the degree of the preimage which results in a $\mathrm{PH}$ curve of a higher degree $[15,23]$. An alternative approach to determine a $\mathrm{PH}$ curve that interpolates the data in a geometric sense was proposed in [14], where a separation of geometric parameters from the coefficients of a curve is right at hand. Moreover, it provides a way to analyse the interpolation problem independently of the dimension of the space. This property is connected to the fact that a PH curve of degree $n$ can interpolate $n+1$ geometric data regardless of the dimension, as was conjectured in [12].

Many interpolation and approximation methods using $\mathrm{PH}$ curves have been developed. Since only odd degree PH curves are regular, cubic and quintic curves are probably the most interesting from the practical point of view. Interpolation schemes using planar cubic PH curves can be found in $[3,12,13,19]$, and spatial PH cubics are considered in $[14,16,17,21]$. For planar quintic PH curves, $C^{2}$ continuous splines that interpolate given points were constructed in [1] and $G^{1}$ Hermite interpolation problem was studied in [4]. Interpolation of $G^{1}$ Hermite data together with end curvatures by monotone helical quintics, which form a subclass of general spatial PH curves, is done in [11].

When designing curves it is often desirable to join two points with a $G^{2}$ contact. Not many results are known for this type of interpolation using PH curves of low degree. A planar cubic $G^{2}$ spline interpolating only the given points is constructed in [13]. A spatial $G^{2}$ continuous curve composed of a pair of PH quintic spiral segments is examined in [20]. For a $G^{2}$ interpolation scheme with PH curves of degree seven see [15]. 
In this paper, we consider the problem of interpolating $G^{2}$ Hermite data in $\mathbb{R}^{d}$, $d \geq 2$, with PH curves with the lowest possible degree, independently of the dimension $d$. More precisely, a $G^{2}$ quintic PH spline that interpolates two points, two tangent directions and two curvature vectors at these points at every polynomial segment is searched for. The problem was exposed as an open problem in [11] for spatial as well as for planar PH curves. The standard approach to this interpolation problem via complex or quaternion preimage is not prosperous. Mostly due to the fact that separation of unknown geometric interpolation parameters and coefficients of the preimage is obviously a hard task. Therefore, to tackle the problem, we follow the approach introduced in [14] and apply it to the above $G^{2}$ Hermite interpolation by PH quintics. We succeed to reduce the problem to a system of two polynomial equations (see Theorem 2.1) which is independent of the space dimension and involves only tangent lengths as unknowns.

One might use some polynomial solver to find all the solutions and apply any standard method to select the most acceptable one according to some criteria, e.g., the smallest absolute rotation index, minimal bending energy (see [8] and the references therein), etc. Here we suggest an alternative approach based on the asymptotic analysis. Numerical examples show that there might exist several solutions which are not qualitatively equivalent. As observed, some do much better as far as asymptotic approximation order and shape is concerned and some are quite unacceptable. This gives an idea for a general algorithm based upon a detailed asymptotic analysis how to single out the desired solution. It starts with a particular problem with a unique best approximation order solution and trace it to a solution of a general problem by a homotopy continuation method [2]. Numerical experiments show that this procedure behaves well in practice.

The paper is organized as follows. In Section 2, the interpolation problem is precisely stated and a system of nonlinear algebraic equations is derived. A preliminary numerical example is given in Section 3 to justify the necessity of asymptotic analysis which is done in detail. The existence of several solutions of different approximation order is established and their asymptotic behaviour is analyzed. Section 4 describes a general algorithm for solving the interpolation problem. A homotopy method tracing an appropriate solution of the particular problem to a solution of the problem for general data is explained in detail. Finally, a numerical example is given which confirms theoretical results.

\section{Interpolation problem}

Suppose that the data

$$
\boldsymbol{f}_{0}, \boldsymbol{d}_{0}, \boldsymbol{P}_{0}, \boldsymbol{P}_{1}, \boldsymbol{d}_{1}, \boldsymbol{f}_{1}
$$

are prescribed in $\mathbb{R}^{d}, d \geq 2$, where the points $\boldsymbol{P}_{i}$, the vectors $\boldsymbol{d}_{i}$ and $\boldsymbol{f}_{i}, i=0,1$, represent the values, the first and the second derivatives of some regular parametric curve, sampled at two distinct parameter values. We will study the following interpolation 
problem: determine a quintic Pythagorean-hodograph curve $\boldsymbol{p}:[0,1] \rightarrow \mathbb{R}^{d}$ that interpolates the prescribed data in a geometric $G^{2}$ sense. The $G^{2}$ interpolation conditions can be written as

$$
\boldsymbol{p}(u)=\boldsymbol{P}_{u}, \quad \boldsymbol{p}^{\prime}(u)=\lambda_{u} \boldsymbol{d}_{u}, \quad \boldsymbol{p}^{\prime \prime}(u)=\lambda_{u}^{2} \boldsymbol{f}_{u}+\mu_{u} \boldsymbol{d}_{u}, \quad u=0,1,
$$

where

$$
\lambda_{i}>0, \quad \mu_{i} \in \mathbb{R}, \quad i=0,1,
$$

are free parameters. In addition, the interpolating curve $\boldsymbol{p}$ should satisfy the $\mathrm{PH}$ condition, i.e., its parametric speed

$$
\left\|\boldsymbol{p}^{\prime}(t)\right\|, \quad t \in[0,1]
$$

should be a polynomial of degree $\leq 4$. Here, $\|\boldsymbol{v}\|:=\sqrt{\boldsymbol{v}^{T} \boldsymbol{v}}$ denotes the Euclidean norm, implied by the standard scalar product.

The first simplification of the interpolation problem is straightforward. If the prescribed data are based upon the arc-length parameterization of the underlying curve, we observe

$$
\left\|\boldsymbol{d}_{i}\right\|=1, \quad \boldsymbol{d}_{i}^{T} \boldsymbol{f}_{i}=0, \quad i=0,1 .
$$

If this is not the case, we may apply the Gram-Schmidt orthogonalization steps

$$
\boldsymbol{f}_{i} \rightarrow \frac{1}{\left\|\boldsymbol{d}_{i}\right\|^{2}}\left(\boldsymbol{f}_{i}-\frac{\boldsymbol{f}_{i}^{T} \boldsymbol{d}_{i}}{\left\|\boldsymbol{d}_{i}\right\|^{2}} \boldsymbol{d}_{i}\right), \quad \boldsymbol{d}_{i} \rightarrow \frac{1}{\left\|\boldsymbol{d}_{i}\right\|} \boldsymbol{d}_{i}, \quad i=0,1,
$$

so that the modified data vectors satisfy (2.2). Note that the interpolation conditions (2.1) remain of the same form if one properly renames the unknowns. Thus we shall assume throughout the paper that the original data already satisfy (2.2).

A proper choice of a basis of the polynomial space $\mathbb{P}_{5}$ gives the interpolating curve that satisfies (2.1) in a simple closed form. With

$$
\begin{aligned}
& \pi_{1}(t):=(1-t)^{3} t(3 t+1), \quad \pi_{2}(t):=\frac{1}{2}(1-t)^{3} t^{2}, \\
& \pi_{3}(t):=t^{3}\left(6 t^{2}-15 t+10\right), \quad \pi_{4}(t):=\pi_{2}(1-t), \quad \pi_{5}(t):=-\pi_{1}(1-t),
\end{aligned}
$$

the curve $\boldsymbol{p}$ reads

$$
\boldsymbol{p}=\boldsymbol{f}_{0} \lambda_{0}^{2} \pi_{2}+\boldsymbol{d}_{0}\left(\lambda_{0} \pi_{1}+\mu_{0} \pi_{2}\right)+\boldsymbol{P}_{0}+\Delta \boldsymbol{P}_{0} \pi_{3}+\boldsymbol{d}_{1}\left(\lambda_{1} \pi_{5}+\mu_{1} \pi_{4}\right)+\boldsymbol{f}_{1} \lambda_{1}^{2} \pi_{4},
$$

where $\Delta \boldsymbol{P}_{0}:=\boldsymbol{P}_{1}-\boldsymbol{P}_{0}$. Thus the linear part (2.1) of the interpolation problem is fulfilled. In order to derive additional equations that correspond to the $\mathrm{PH}$ condition, we follow [14, Sec. 3]. Let us define

$$
\boldsymbol{w}:=\left(\lambda_{0}^{2} \pi_{2}^{\prime}, \lambda_{0} \pi_{1}^{\prime}+\mu_{0} \pi_{2}^{\prime}, \pi_{3}^{\prime}, \lambda_{1} \pi_{5}^{\prime}+\mu_{1} \pi_{4}^{\prime}, \lambda_{1}^{2} \pi_{4}^{\prime}\right)^{T},
$$

and

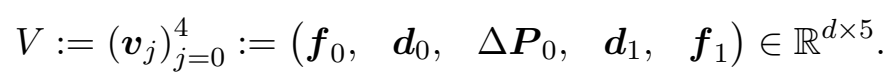


The hodograph can be expressed as

$$
p^{\prime}=V w
$$

and the parametric speed is given by

$$
\left\|\boldsymbol{p}^{\prime}\right\|=\sqrt{\boldsymbol{w}^{T} G \boldsymbol{w}}, \quad G:=V^{T} V .
$$

The Gram matrix $G$ is a symmetric positive semidefinite matrix that depends on the given data only. The assumption (2.2) then simplifies the Gram matrix to

$$
G=\left(\begin{array}{ccccc}
g_{00} & 0 & g_{02} & g_{03} & g_{04} \\
0 & 1 & g_{12} & g_{13} & g_{14} \\
g_{02} & g_{12} & g_{22} & g_{23} & g_{24} \\
g_{03} & g_{13} & g_{23} & 1 & 0 \\
g_{04} & g_{14} & g_{24} & 0 & g_{44}
\end{array}\right)
$$

There are several approaches on how to deal with PH curves. For planar curves one would usually use complex representation, for spatial ones quaternion algebra is involved. For curves of higher degree a generalization of these structures might be used leading to Clifford algebras. But there is also a straightforward way to incorporate $\mathrm{PH}$ conditions in the interpolation problem, i.e., to write them in a purely algebraic way. Here we found this approach the most promising one, since it led to the simplest set of nonlinear equations independently of the space dimension $d$. In order to apply it, let us recall $[14$, Rem. 1$]$, with a particular choice of the points, divided differences [.] are based upon. The parametric speed (2.3) would be a polynomial of degree $\leq 4$ iff the following (scaled) equations

$$
e_{i}:=e_{i}\left(\lambda_{0}, \lambda_{1}, \mu_{0}, \mu_{1}\right):=\frac{1}{c_{i}}[\underbrace{0, \cdots, 0}_{i+1}, \underbrace{1, \cdots, 1}_{5-i}]\left\|\boldsymbol{p}^{\prime}(\cdot)\right\|=0, \quad i=0,1, \cdots, 4,
$$

are satisfied, where constants $c_{i}, i=0,1, \cdots, 4$, are equal to $8,2,2,2,8$ respectively. Four equations are independent only, and one may take either the first equation $e_{0}=0$ or the last one $e_{4}=0$ as extraneous. It is straightforward to compute the polynomials $\lambda_{1} e_{0}, e_{1}, e_{2}, e_{3}, \lambda_{0} e_{4} \in \mathbb{R}\left[\lambda_{0}, \lambda_{1}, \mu_{0}, \mu_{1}\right]$,

$$
\begin{aligned}
e_{1}= & \lambda_{1}\left(\lambda_{1} g_{44}\left(7 \lambda_{1}-\mu_{1}\right)+60 g_{24}\right)-\mu_{0}\left(3 \lambda_{1} g_{14}+2 g_{13}-2\right) \\
& -\lambda_{0}^{2}\left(3 \lambda_{1} g_{04}+2 g_{03}\right)-8 \lambda_{0}\left(3 \lambda_{1} g_{14}+g_{13}-1\right), \\
e_{2}= & g_{44} \lambda_{1}^{3}-g_{00} \lambda_{0}^{3}-3\left(g_{03} \lambda_{0}^{2}+g_{14} \lambda_{1}^{2}\right)+24\left(\lambda_{0}-\lambda_{1}\right)\left(1-g_{13}\right) \\
& +3\left(\left(\mu_{0}+\mu_{1}\right)\left(1-g_{13}\right)-20 g_{12}+20 g_{23}\right) \\
e_{3}= & \lambda_{0}\left(60 g_{02}-\lambda_{0} g_{00}\left(7 \lambda_{0}+\mu_{0}\right)\right)+\mu_{1}\left(3 \lambda_{0} g_{03}-2 g_{13}+2\right) \\
& +\lambda_{1}^{2}\left(3 \lambda_{0} g_{04}-2 g_{14}\right)-8 \lambda_{1}\left(3 \lambda_{0} g_{03}-g_{13}+1\right),
\end{aligned}
$$




$$
\begin{aligned}
e_{4}= & 4 g_{03}\left(3 \mu_{0} \mu_{1}-140 \lambda_{0} \lambda_{1}\right)+\lambda_{0}^{3} g_{00}\left(\lambda_{0}^{2} g_{00}-169\right)+240 \mu_{0} g_{02} \\
& +2\left(\lambda_{1}^{2} g_{04}\left(37 \lambda_{0}+6 \mu_{0}\right)+\lambda_{0}\left(-2 \mu_{0}^{2} g_{00}+37 \mu_{1} g_{03}+660 g_{02}\right)\right) \\
& +2 \lambda_{0}^{2} g_{00}\left(3 g_{13}\left(\mu_{1}-8 \lambda_{1}\right)+60 g_{12}-25 \mu_{0}\right)-8 \lambda_{1}\left(12 \mu_{0} g_{03}+g_{13}-1\right) \\
+ & \frac{1}{\lambda_{0}}\left(6 \lambda_{1}\left(\lambda_{1} g_{14}\left(\lambda_{0}^{3} g_{00}-24 \lambda_{1} g_{13}\right)+24 \mu_{1}\left(1-g_{13}^{2}\right)-480\left(g_{12} g_{13}-g_{23}\right)\right)\right. \\
& +9\left(\mu_{1}^{2}\left(g_{13}^{2}-1\right)+40 \mu_{1}\left(g_{12} g_{13}-g_{23}\right)+400\left(g_{12}^{2}-g_{22}\right)\right) \\
& \left.+9 \lambda_{1}^{2}\left(\lambda_{1}^{2}\left(g_{14}^{2}-g_{44}\right)+2 \mu_{1} g_{13} g_{14}+64 g_{13}^{2}+40 g_{12} g_{14}-40 g_{24}-64\right)\right),
\end{aligned}
$$

and similarly $e_{0}$. Note that the first three polynomials in (2.4) are linear in the variables $\mu_{0}$ and $\mu_{1}$. If we express them from $e_{1}$ and $e_{3}$, we obtain

$$
\begin{aligned}
& \mu_{0}\left(\lambda_{0}, \lambda_{1}\right)=\frac{\left(2-2 g_{13}+3 g_{03} \lambda_{0}\right) e_{1}\left(\lambda_{0}, \lambda_{1}, 0,0\right)+g_{44} \lambda_{1}^{2} e_{3}\left(\lambda_{0}, \lambda_{1}, 0,0\right)}{D\left(\lambda_{0}, \lambda_{1}\right)}, \\
& \mu_{1}\left(\lambda_{0}, \lambda_{1}\right)=\frac{\left(2-2 g_{13}-3 g_{14} \lambda_{1}\right) e_{3}\left(\lambda_{0}, \lambda_{1}, 0,0\right)+g_{00} \lambda_{0}^{2} e_{1}\left(\lambda_{0}, \lambda_{1}, 0,0\right)}{D\left(\lambda_{0}, \lambda_{1}\right)},
\end{aligned}
$$

with the denominator given as

$$
D\left(\lambda_{0}, \lambda_{1}\right):=g_{00} g_{44} \lambda_{0}^{2} \lambda_{1}^{2}+\left(3 g_{03} \lambda_{0}-2 g_{13}+2\right)\left(3 g_{14} \lambda_{1}+2 g_{13}-2\right) .
$$

Further, if we insert the expressions (2.5) in the remaining two equations, we end up with a system of two rational equations

$$
e_{i}\left(\lambda_{0}, \lambda_{1}, \mu_{0}\left(\lambda_{0}, \lambda_{1}\right), \mu_{1}\left(\lambda_{0}, \lambda_{1}\right)\right)=0, \quad i=2,4
$$

for the unknowns $\lambda_{0}$ and $\lambda_{1}$. The polynomial form of (2.7) is given as

$$
\begin{aligned}
& D\left(\lambda_{0}, \lambda_{1}\right) e_{2}\left(\lambda_{0}, \lambda_{1}, \mu_{0}\left(\lambda_{0}, \lambda_{1}\right), \mu_{1}\left(\lambda_{0}, \lambda_{1}\right)\right)=0, \\
& D\left(\lambda_{0}, \lambda_{1}\right)^{2} \lambda_{0} e_{4}\left(\lambda_{0}, \lambda_{1}, \mu_{0}\left(\lambda_{0}, \lambda_{1}\right), \mu_{1}\left(\lambda_{0}, \lambda_{1}\right)\right)=0 .
\end{aligned}
$$

Let us summarize the preceding discussion.

Theorem 2.1. Any pair $\lambda_{0}>0, \lambda_{1}>0$ that satisfies polynomial equations (2.8), such that $D\left(\lambda_{0}, \lambda_{1}\right) \neq 0$, determines the quintic $P H$ curve that solves the interpolation problem (2.1) with the data that satisfy (2.2). Corresponding $\mu_{0}, \mu_{1}$ are computed from (2.5).

The equations (2.8) are of the total degree 7 and 14 respectively, quite in reach of nowadays polynomial equations solvers. However, since several solutions are to be expected, a robust recipe that would usually give the proper solution would be appropriate.

Quite clearly, a change of sign of the data vectors, and the corresponding reparameterization $t \rightarrow 1-t$ of the curve $\boldsymbol{p}$ should lead to a system that evolves from (2.4). The following remark reflects this. 
Remark 2.1. Suppose that the elements of the Gram matrix $G$ are changed as follows,

$$
\begin{aligned}
& g_{00} \rightarrow g_{44}, \quad g_{44} \rightarrow g_{00}, \quad g_{02} \rightarrow-g_{24}, \quad g_{24} \rightarrow-g_{02}, \\
& g_{03} \rightarrow g_{14}, \quad g_{14} \rightarrow g_{03}, \quad g_{12} \rightarrow-g_{23}, \quad g_{23} \rightarrow-g_{12} \text {. }
\end{aligned}
$$

If we change the unknowns

$$
\lambda_{0} \rightarrow-\lambda_{1}, \quad \lambda_{1} \rightarrow-\lambda_{0}, \quad \mu_{0} \rightarrow \mu_{1}, \quad \mu_{1} \rightarrow \mu_{0},
$$

too, we obtain the same system of equations (2.4), since

$$
e_{5} \rightarrow e_{0}, \quad e_{0} \rightarrow e_{5}, \quad e_{3} \rightarrow e_{1}, \quad e_{1} \rightarrow e_{3}
$$

\section{Asymptotic analysis}

Not all solutions of Eq. (2.7) that satisfy $\lambda_{0}>0, \lambda_{1}>0$ are acceptable from the approximation point of view. One would expect that the interpolation curve $\boldsymbol{p}$ would be an approximation of order six, at least for moderate data. As an example, let us consider a particular curve $\boldsymbol{g}:[0, b] \rightarrow \mathbb{R}^{3}$,

$$
\boldsymbol{g}(t):=\frac{1}{\sqrt{5}}\left(\begin{array}{c}
\sqrt{5} \ln (1+t) \cos t \\
\sqrt{1+t^{2}}-1+2 \ln (1+t) \sin t \\
2\left(\sqrt{1+t^{2}}-1\right)-\ln (1+t) \sin t
\end{array}\right)
$$

interpolated at $t=0$ and $t=b$.

A choice $b=\frac{1}{4}$ yields five admissible solutions (see Fig. 1). One of them is obviously unacceptable, and there is an undesirable kink in the other solution too. The remaining three, which are shown in a better local view in Fig. 2 seem to be similar at a first glance. So we are faced with a question, discussed quite often in the literature (see, e.g., $[1,4,9])$ : which solution should be returned by a computer program as the most

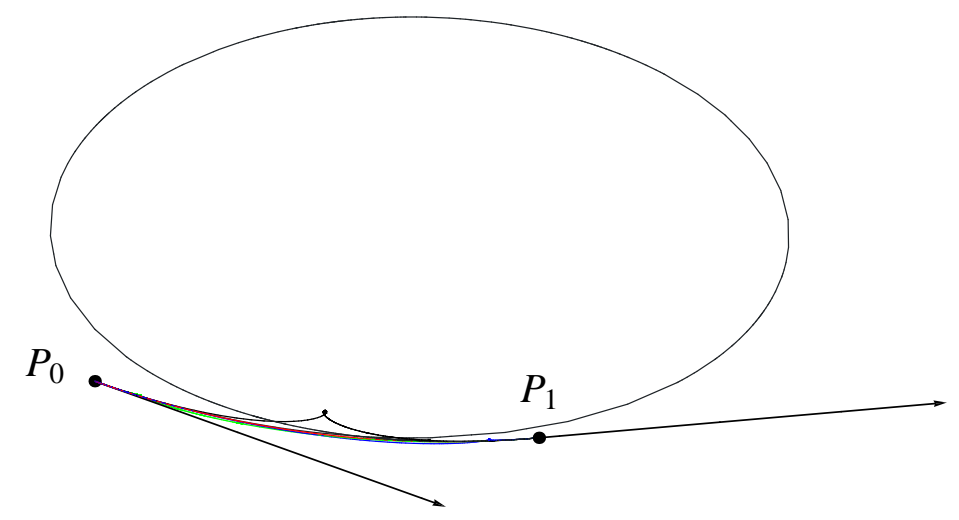

Figure 1: Five solutions of a particular $G^{2}$ quintic interpolation problem. 


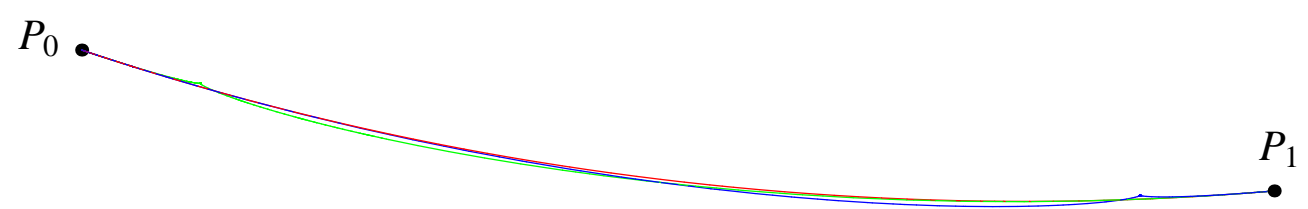

Figure 2: The three best solutions of a particular $G^{2}$ quintic interpolation problem.

appropriate one? But the data here are provided by the known curve $\boldsymbol{g}$, and some further analysis can be carried out. Table 1 reveals the Hausdorff distance between $\boldsymbol{g}$ and the interpolating curves. Quite clearly, the first solution is the one to be chosen. However, since this error estimate selection is not computable in the general case, let us trace the solutions obtained as functions of decreasing $b$. If the Hausdorff distance decays as $\approx$ const $b^{-\alpha}$, the decay rate $\alpha$ may be estimated from Hausdorff distances computed for consecutive $b$-s. The result of the numerical experiment, Fig. 3, indicates that the solutions belong to three entirely different approximation order classes: $\alpha \approx$ $0,2,6$. This gives a motivation for a rigorous asymptotic analysis as well as a hint for a numerical algorithm that gives an appropriate solution of Eq. (2.7): a continuation method (e.g., [2]) that begins with a model problem and its particular solution of the approximation order six.

Table 1: The Hausdorff distance and parameters $\lambda_{i}, \mu_{i}, i=0,1$, with $b=\frac{1}{4}$ for all five solutions of a particular $G^{2}$ quintic interpolation problem.

\begin{tabular}{||c|c|c|c|c|c||}
\hline & Hausdorff distance & $\frac{1}{b} \lambda_{0}$ & $\frac{1}{b} \lambda_{1}$ & $\frac{1}{b} \mu_{0}$ & $\frac{1}{b} \mu_{1}$ \\
\hline 1 & $1.23236 \times 10^{-6}$ & 1.03695 & 0.86432 & -0.40493 & 0.036217 \\
2 & $1.45516 \times 10^{-3}$ & 1.27367 & 2.27877 & -11.1875 & 1.69003 \\
3 & $1.41561 \times 10^{-3}$ & 2.51591 & 1.16926 & -3.09427 & 9.94449 \\
4 & $1.71950 \times 10^{-2}$ & 3.02391 & 2.68586 & -13.1529 & 11.2369 \\
5 & $3.51152 \times 10^{-1}$ & 2.62773 & 2.32101 & -13.2049 & 11.1413 \\
\hline
\end{tabular}

In order to analyse the asymptotic behaviour of the solutions $\lambda_{i}$ and $\mu_{i}$, let us assume that the data are sampled from a smooth parametric curve $r:\left[-\frac{h}{2}, \frac{h}{2}\right] \rightarrow \mathbb{R}^{d}, 2 \leq$ $d$, parameterized by the arc-length $s$. Although we have to choose $d=5$ in order to avoid possible irregular PH solution [14, Lemma 2], for the asymptotic analysis it does not really matter which particular dimension $d$ one selects. The analysis will therefore be given for the most natural choice, i.e., $d=3$. The data to be interpolated are

$$
\begin{array}{llll}
\boldsymbol{P}_{0} & =\boldsymbol{r}\left(-\frac{h}{2}\right), & \boldsymbol{d}_{0}=\boldsymbol{r}^{\prime}\left(-\frac{h}{2}\right), & \boldsymbol{f}_{0}=\boldsymbol{r}^{\prime \prime}\left(-\frac{h}{2}\right), \\
\boldsymbol{P}_{1}=\boldsymbol{r}\left(\frac{h}{2}\right), & \boldsymbol{d}_{1}=\boldsymbol{r}^{\prime}\left(\frac{h}{2}\right), & \boldsymbol{f}_{1}=\boldsymbol{r}^{\prime \prime}\left(\frac{h}{2}\right),
\end{array}
$$

where the interval length $h$ is assumed to be as small as needed. Suppose also that the Frenet frame of $\boldsymbol{r}$, denoted $\mathcal{F}(\boldsymbol{r})$, is well defined for all $s \in\left[-\frac{h}{2}, \frac{h}{2}\right]$. Without loss of 


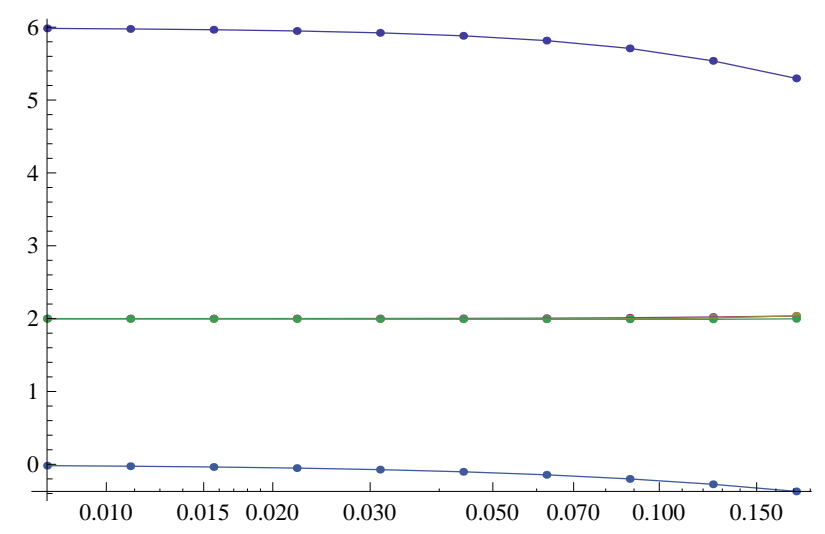

Figure 3: Decay rates of the Hausdorff distance between $\boldsymbol{g}$ and the five polynomial interpolants depending on the interval length $b$. The solutions of decay 2 are almost indistinguishable.

generality we may choose the coordinate system with the origin at $\boldsymbol{r}(0)$, and the axes determined by the Frenet frame of $r$ at $t=0$, which somewhat simplifies the expansion of $\boldsymbol{r}$. Suppose that the curvature $\kappa$ and the torsion $\tau$ of the curve $\boldsymbol{r}$ at $s=0$ expand as

$$
\kappa(s)=\kappa_{0}+\frac{\kappa_{1}}{1 !} s+\frac{\kappa_{2}}{2 !} s^{2}+\frac{\kappa_{3}}{3 !} s^{3}+\mathcal{O}\left(s^{4}\right), \quad \tau(s)=\tau_{0}+\frac{\tau_{1}}{1 !} s+\frac{\tau_{2}}{2 !} s^{2}+\frac{\tau_{3}}{3 !} s^{3}+\mathcal{O}\left(s^{4}\right),
$$

where the fact $\kappa_{0}>0$ will be needed throughout this section. Since $\boldsymbol{r}^{\prime}=(\mathcal{F}(\boldsymbol{r}))_{1}$, and we have assumed

$$
\boldsymbol{r}(0)=(0,0,0)^{T}, \quad \mathcal{F}(\boldsymbol{r})(0)=\left(\delta_{i, j}\right)_{i, j=1}^{3},
$$

it is straightforward to derive the expansion of $\boldsymbol{r}$,

$$
\boldsymbol{r}(s)=\boldsymbol{r}(0)+\boldsymbol{r}^{\prime}(0) \frac{1}{1 !} s+\boldsymbol{r}^{\prime \prime}(0) \frac{1}{2 !} s^{2}+\cdots,
$$

from the Frenet-Serret formulas as

$$
\boldsymbol{r}(s)=\left(\begin{array}{l}
s-\frac{1}{6} \kappa_{0}^{2} s^{3}-\frac{1}{8} \kappa_{0} \kappa_{1} s^{4}+\mathcal{O}\left(s^{5}\right) \\
\frac{1}{2} \kappa_{0} s^{2}+\frac{1}{6} \kappa_{1} s^{3}-\frac{1}{24}\left(\kappa_{0}^{3}+\tau_{0}^{2} \kappa_{0}-\kappa_{2}\right) s^{4}+\mathcal{O}\left(s^{5}\right) \\
\frac{1}{6} \kappa_{0} \tau_{0} s^{3}+\frac{1}{24}\left(2 \kappa_{1} \tau_{0}+\kappa_{0} \tau_{1}\right) s^{4}+\mathcal{O}\left(s^{5}\right)
\end{array}\right)
$$

With a help of (3.3) we obtain expansions of the asymptotic data in (3.2). The elements $g_{i j}:=g_{i j}(h)$ of the Gram matrix expand as

$$
\begin{aligned}
& g_{00}(h)=\kappa_{0}^{2}-\kappa_{0} \kappa_{1} h+\frac{1}{4}\left(\kappa_{0} \kappa_{2}+\kappa_{1}^{2}\right) h^{2}-\frac{1}{24}\left(\kappa_{0} \kappa_{3}+3 \kappa_{1} \kappa_{2}\right) h^{3}+\mathcal{O}\left(h^{4}\right), \\
& g_{22}(h)=h^{2}-\frac{1}{12} \kappa_{0}^{2} h^{4}+\mathcal{O}\left(h^{6}\right), \quad g_{44}(h)=g_{00}(-h),
\end{aligned}
$$


and

$$
\begin{aligned}
& g_{02}(h)=\frac{1}{2} \kappa_{0}^{2} h^{2}-\frac{1}{3} \kappa_{0} \kappa_{1} h^{3}+\mathcal{O}\left(h^{4}\right), \\
& g_{03}(h)=\kappa_{0}^{2} h-\frac{1}{2} \kappa_{0} \kappa_{1} h^{2}-\frac{1}{6} \kappa_{0}\left(\kappa_{0}^{3}+\kappa_{0} \tau_{0}^{2}-\kappa_{2}\right) h^{3}+\mathcal{O}\left(h^{4}\right), \\
& g_{04}(h)=\kappa_{0}^{2}+\frac{1}{4}\left(\kappa_{0}\left(\kappa_{2}-2 \kappa_{0}\left(\kappa_{0}^{2}+\tau_{0}^{2}\right)\right)-\kappa_{1}^{2}\right) h^{2}+\mathcal{O}\left(h^{4}\right), \\
& g_{12}(h)=h-\frac{1}{6} \kappa_{0}^{2} h^{3}+\mathcal{O}\left(h^{4}\right), \\
& g_{13}(h)=1-\frac{1}{2} \kappa_{0}^{2} h^{2}+\mathcal{O}\left(h^{4}\right), \\
& g_{14}(h)=g_{03}(-h), \quad g_{23}(h)=-g_{12}(-h), \quad g_{24}(h)=-g_{02}(-h) .
\end{aligned}
$$

A brief look at Remark 2.1, and the expansions (3.4) and (3.5) confirm the following lemma.

Lemma 3.1. The asymptotic form of the system (2.4) has a solution

$$
\lambda_{0}=\lambda_{0}(h), \quad \lambda_{1}=\lambda_{1}(h), \quad \mu_{0}=\mu_{0}(h), \quad \mu_{1}=\mu_{1}(h)
$$

for $h$ small enough if and only if

$$
\lambda_{0}=-\lambda_{1}(-h), \quad \lambda_{1}=-\lambda_{0}(-h), \quad \mu_{0}=\mu_{1}(h), \quad \mu_{1}=\mu_{0}(-h)
$$

is a solution too.

As $h \rightarrow 0$, the parameters $\lambda_{i}$ of a regular curve should stay positive and bounded, so we may assume that they behave as

$$
\lambda_{0}=\xi_{0} h^{\alpha}+\mathcal{O}\left(h^{\alpha}\right), \lambda_{1}=\xi_{1} h^{\beta}+\mathcal{O}\left(h^{\beta}\right), \quad \xi_{0}>0, \xi_{1}>0, \quad \alpha \geq 0, \beta \geq 0 .
$$

Lemma 3.2. Suppose that $\kappa_{0} \neq 0$, and $h>0$ is small enough. Further, let the unknowns $\lambda_{i}$ expand as (3.6). The only possible exponents $\alpha, \beta$ are

$$
\alpha=\beta=0,
$$

or

$$
\alpha=\beta=1 \text {. }
$$

Proof. Let us insert the expansions (3.4) and (3.5) in the polynomial form of the system (2.8). The first equation $e_{2}$ expands as

$$
\begin{aligned}
e_{2}= & \kappa_{0}^{6} \lambda_{0}^{2} \lambda_{1}^{2}\left(\lambda_{1}^{3}-\lambda_{0}^{3}\right)(1+\mathcal{O}(h))+3 \kappa_{0}^{6} \lambda_{0}^{2} \lambda_{1}^{2}\left(\lambda_{1}^{2}-\lambda_{0}^{2}\right)\left(h+\mathcal{O}\left(h^{2}\right)\right) \\
& +\frac{3}{2} \kappa_{0}^{6} \lambda_{0} \lambda_{1}\left(\lambda_{0}-\lambda_{1}\right)\left(3 \lambda_{0}^{2}+4 \lambda_{1} \lambda_{0}+3 \lambda_{1}^{2}\right)\left(h^{2}+\mathcal{O}\left(h^{3}\right)\right) \\
& +21 \kappa_{0}^{5} \lambda_{0} \lambda_{1}\left(\lambda_{0}^{2}-\lambda_{1}^{2}\right)\left(h^{3}+\mathcal{O}\left(h^{4}\right)\right)
\end{aligned}
$$


Table 2: The dominating terms and the corresponding dominance region.

\begin{tabular}{||c|c||}
\hline term & dominance region \\
\hline $39 \kappa_{0}^{6} \xi_{0} h^{\alpha+6}$ & $1<\alpha<2 \wedge \beta>\alpha$ \\
$-39 \kappa_{0}^{6} \xi_{1} h^{\beta+6}$ & $\beta>1 \wedge((\alpha>1 \wedge \beta<\alpha \wedge \alpha \leq 2) \vee(\alpha>2 \wedge \beta<2))$ \\
$-\frac{7}{2} \kappa_{0}^{6} \xi_{0}^{3} h^{3 \alpha+4}$ & $0 \leq \alpha<1 \wedge \alpha+\beta>2$ \\
$\frac{7}{2} \kappa_{0}^{6} \xi_{1}^{3} h^{3 \beta+4}$ & $\beta<1 \wedge((\alpha+\beta>2 \wedge \alpha>1 \wedge \alpha \leq 2) \vee(\alpha>2 \wedge \beta \geq 0))$ \\
$-\kappa_{0}^{6} \xi_{0}^{5} \xi_{1}^{2} h^{5 \alpha+2 \beta}$ & $\alpha \geq 0 \wedge \alpha<\beta \wedge \alpha+\beta<2$ \\
$\kappa_{0}^{6} \xi_{0}^{2} \xi_{1}^{5} h^{2 \alpha+5 \beta}$ & $(\alpha \leq 1 \wedge 0 \leq \beta<\alpha) \vee(\alpha>1 \wedge \beta \geq 0 \wedge \alpha+\beta<2)$ \\
$-\frac{1}{2} \kappa_{0}^{5}\left(\kappa_{0}\left(\lambda_{0}-\lambda_{1}\right)\left(7 \lambda_{0}^{2}+88 \lambda_{1} \lambda_{0}+7 \lambda_{1}^{2}\right)+10 \kappa_{1} \lambda_{0} \lambda_{1}\right)\left(h^{4}+\mathcal{O}\left(h^{5}\right)\right)$ \\
$-18 \kappa_{0}^{6}\left(\lambda_{0}^{2}-\lambda_{1}^{2}\right)\left(h^{5}+\mathcal{O}\left(h^{6}\right)\right)+39 \kappa_{0}^{6}\left(\lambda_{0}-\lambda_{1}\right)\left(h^{6}+\mathcal{O}\left(h^{7}\right)\right)$ \\
$+5 \kappa_{0}^{5}\left(\kappa_{1}+\frac{3}{4}\left(\lambda_{0} \lambda_{1} \tau_{0}\left(\kappa_{1} \tau_{0}-\kappa_{0} \tau_{1}\right)\right)\right)\left(h^{8}+\mathcal{O}\left(h^{9}\right)\right)$.
\end{tabular}

If we insert (3.6) in (3.9), we obtain a sum of terms with various exponents of $h$, depending on $\alpha$ and $\beta$. Six of the terms, each of them in a particular subregion of $\{(\alpha, \beta) \mid \alpha \geq 0, \beta \geq 0\}$ respectively, become dominant as $h \rightarrow 0$ (Table 2). This leaves us with the following possible exponents

$$
\begin{aligned}
& 0 \leq \alpha=\beta<2, \\
& \alpha+\beta=2 \wedge \alpha \geq 0 \wedge \beta \geq 0, \\
& \alpha=1 \wedge \beta>1, \quad \beta=1 \wedge \alpha>1, \quad \alpha \geq 2 \wedge \beta \geq 2,
\end{aligned}
$$

which could not be excluded by a single dominating term (Fig. 4). The expansion of the second equation $e_{4}$ is rather long. But the particular case simplification

$$
e_{4}=-900 \kappa_{10}^{10} h^{12}+\mathcal{o}\left(h^{12}\right), \quad \alpha>1 \wedge \beta>1,
$$

rules out the last possibility in (3.12). It also shortens (3.10) to $0 \leq \alpha=\beta \leq 1$. In the latter case, if $\alpha \in(0,1)$, the equations expand as

$$
\begin{aligned}
& e_{2}=-\kappa_{0}^{6} \xi_{0}^{2} \xi_{1}^{2}\left(\xi_{0}^{3}-\xi_{1}^{3}\right) h^{7 \alpha}+\mathcal{o}\left(h^{7 \alpha}\right), \\
& e_{4}=-\kappa_{0}^{10} \xi_{0}^{4} \xi_{1}^{3}\left(18 \xi_{0}^{5}+15 \xi_{1} \xi_{0}^{4}+6 \xi_{1}^{2} \xi_{0}^{3}-8 \xi_{1}^{3} \xi_{0}^{2}+9 \xi_{1}^{5}\right)+\mathcal{o}\left(h^{12 \alpha}\right) .
\end{aligned}
$$

The leading terms can not both vanish since the leading one of $e_{2}$ implies $\xi_{1}=\xi_{0}$, but then

$$
e_{4}=-40 \kappa_{0}^{10} \xi_{0}^{12}+\mathcal{o}\left(h^{12 \alpha}\right) .
$$

This proves that the only possible exponents in the case (3.10) are given by (3.7). Let us consider now the case (3.11). The main part of the expansion reads

$$
\begin{aligned}
& e_{4}=6 \xi_{0}^{7}\left(1-\xi_{0} \xi_{1}\right)\left(2 \xi_{0} \xi_{1}-7\right) \kappa_{0}^{10} h^{7 \alpha+5}+\mathcal{o}\left(h^{7 \alpha+5}\right), \quad 0 \leq \alpha<1, \\
& e_{4}=-\kappa_{0}^{10} \xi_{1}^{6}\left(9-4 \xi_{0} \xi_{1}\right)^{2}+\mathcal{o}\left(h^{18-6 \alpha}\right), \quad 1<\alpha \leq 2 .
\end{aligned}
$$




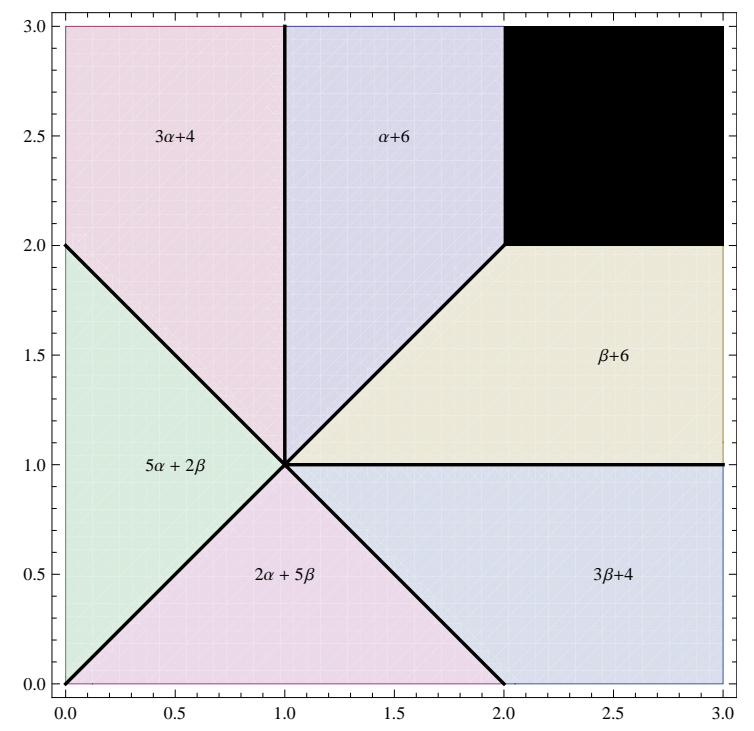

Figure 4: Exponents of the dominating terms and the corresponding dominance region. The part of the first quadrant $\alpha \geq 0, \beta \geq 0$ that can not be excluded is shown in black.

Both leading terms in (3.13) may vanish. However, these particular cases give

$$
\begin{array}{ll}
\xi_{1}=\frac{1}{\xi_{0}}: e_{4}=-25 \kappa_{0}^{10} \xi_{0}^{6} h^{6 \alpha+6}+\mathcal{o}\left(h^{6 \alpha+6}\right), & 0 \leq \alpha<1, \\
\xi_{1}=\frac{2}{7 \xi_{0}}: e_{4}=200 \kappa_{0}^{10} \xi_{0}^{6} h^{6 \alpha+6}+\mathcal{o}\left(h^{6 \alpha+6}\right), & 0 \leq \alpha<1, \\
\xi_{1}=\frac{9}{4 \xi_{0}}: e_{4}=-\frac{178623225 \kappa_{0}^{10} h^{16-4 \alpha}}{65536 \xi_{0}^{4}}+\mathcal{o}\left(h^{16-4 \alpha}\right), & 1<\alpha \leq 2,
\end{array}
$$

which eliminates all the possible $\alpha$ but $\alpha=1$. Finally, the expansions

$$
\begin{aligned}
& e_{2}=\frac{1}{2} \kappa_{0}^{6} \xi_{1}\left(\xi_{1}\left(7 \xi_{1}+36\right)-78\right) h^{7}+\mathcal{o}\left(h^{7}\right), \quad \alpha>1, \quad \beta=1, \\
& e_{4}=-9 \kappa_{0}^{10}\left(3 \xi_{1}+1\right)^{2}\left(\xi_{1}\left(\xi_{1}+4\right)-10\right)^{2}+\mathcal{o}\left(h^{12}\right), \quad \alpha>1, \quad \beta=1,
\end{aligned}
$$

eliminate the possibility $\alpha>1, \beta=1$, since the leading terms could not vanish simultaneously. The case $\alpha=1, \beta>1$ follows similarly. The proof is completed.

Lemma 3.3. Let the assumptions of Lemma 3.2 be fulfilled. If $\alpha=\beta=0$, there exists a unique solution of (2.4) and the unknowns $\lambda_{i}$ and $\mu_{i}$ expand as

$$
\begin{array}{ll}
\lambda_{0}=\frac{2 \sqrt{10}}{\kappa_{0}}+\frac{2 \sqrt{10} \kappa_{1}}{3 \kappa_{0}^{2}} h+\mathcal{O}\left(h^{2}\right), & \lambda_{1}=\frac{2 \sqrt{10}}{\kappa_{0}}-\frac{2 \sqrt{10} \kappa_{1}}{3 \kappa_{0}^{2}} h+\mathcal{O}\left(h^{2}\right), \\
\mu_{0}=-\frac{8 \sqrt{10}}{\kappa_{0}}+\mathcal{O}(h), & \mu_{1}=\frac{8 \sqrt{10}}{\kappa_{0}}+\mathcal{O}(h) .
\end{array}
$$


Proof. At the limit $h=0$, the unknowns $\lambda_{0}=\xi_{0}, \lambda_{1}=\xi_{1}$ should satisfy the equations $e_{2}=0, e_{4}=0$ when $h=0$. This gives

$$
\left.e_{2}\right|_{h=0}=\pi_{1}\left(\xi_{0}, \xi_{1}\right)=0,\left.\quad e_{4}\right|_{h=0}=\pi_{2}\left(\xi_{0}, \xi_{1}\right)=0,
$$

where

$$
\begin{aligned}
& \pi_{1}\left(\xi_{0}, \xi_{1}\right):=\kappa_{0}^{6} \xi_{0}^{2} \xi_{1}^{2}\left(\xi_{1}^{3}-\xi_{0}^{3}\right), \\
& \pi_{2}\left(\xi_{0}, \xi_{1}\right):=\kappa_{0}^{10} \xi_{0}^{4} \xi_{1}^{3}\left(\kappa_{0}^{2} \xi_{1} \xi_{0}^{6}-18 \xi_{0}^{5}-15 \xi_{1} \xi_{0}^{4}-6 \xi_{1}^{2} \xi_{0}^{3}+8 \xi_{1}^{3} \xi_{0}^{2}-9 \xi_{1}^{5}\right) .
\end{aligned}
$$

The first polynomial of the Gröbner basis of the ideal $\left\langle\pi_{1}, \pi_{2}\right\rangle$ with respect to variables $\left(\xi_{0}, \xi_{1}\right)$ is determined as

$$
\kappa_{0}^{10} \xi_{0}^{2} \xi_{1}^{12}\left(\kappa_{0}^{2} \xi_{1}^{2}-40\right)\left(\kappa_{0}^{4} \xi_{1}^{4}-5 \kappa_{0}^{2} \xi_{1}^{2}+25\right)
$$

This polynomial has precisely one positive root $\xi_{1}=\frac{2 \sqrt{10}}{\kappa_{0}}$, and the rest of the basis vanishes at $\xi_{0}=\frac{2 \sqrt{10}}{\kappa_{0}}$. This gives the limit solutions $\lambda_{i}=\xi_{i}, i=1,2$. The Jacobian at the limit solution is nonsingular, since its determinant equals

$$
-\frac{3145728000000000 \sqrt{10}}{\kappa_{0}}
$$

But then the Implicit function theorem implies that the unknowns $\lambda_{i}$ admit the Taylor series expansion,

$$
\lambda_{0}=\frac{2 \sqrt{10}}{\kappa_{0}}+\zeta_{0} h+\mathcal{O}\left(h^{2}\right), \quad \lambda_{1}=\frac{2 \sqrt{10}}{\kappa_{0}}+\zeta_{1} h+\mathcal{O}\left(h^{2}\right) .
$$

If we insert (3.15) in the expansions of $e_{2}$ and $e_{4}$, the $\mathcal{O}(h)$ terms determine the constants $\zeta_{0}$ and $\zeta_{1}$, which confirms the first row of (3.14). The second one follows then from the expansion of (2.6).

Let us consider now the second possible exponent choice (3.8), i.e., $\alpha=\beta=1$. The leading terms of equations turn out rather long. If we compute the Gröbner basis of both with respect to variables $\left(\xi_{0}, \xi_{1}\right)$, the first basis function, independent of $\xi_{0}$, turns out as

$$
\kappa_{0}^{10}\left(\xi_{1}-1\right)^{9}\left(\xi_{1}-3\right)\left(\xi_{1}^{2}-3 \xi_{1}-1\right)^{2} \pi_{3}\left(\xi_{1}\right) \pi_{4}\left(\xi_{1}\right)^{2} \pi_{5}\left(\xi_{1}\right) h^{12}
$$

where

$$
\begin{aligned}
\pi_{3}\left(\xi_{1}\right):= & \left(\xi_{1}+1\right)^{3}\left(\xi_{1}^{2}+8 \xi_{1}+1\right)^{3}\left(2 \xi_{1}^{2}+8 \xi_{1}+15\right) \\
\pi_{4}\left(\xi_{1}\right):= & 4 \xi_{1}^{10}+51 \xi_{1}^{9}+256 \xi_{1}^{8}+618 \xi_{1}^{7}+2506 \xi_{1}^{6}+2025 \xi_{1}^{5} \\
& +5612 \xi_{1}^{4}-22578 \xi_{1}^{3}-29560 \xi_{1}^{2}-12003 \xi_{1}-1606 \\
\pi_{5}\left(\xi_{1}\right):= & \xi_{1}^{6}-28 \xi_{1}^{5}+823 \xi_{1}^{4}-1236 \xi_{1}^{3}+2478 \xi_{1}^{2}-19008 \xi_{1}+20736 .
\end{aligned}
$$


The polynomial $\pi_{3}$ has obviously no positive roots. The only positive root $\xi_{1} \approx 1.7811$ of the polynomial $\pi_{4}$ determines $\xi_{0} \approx-0.316622$ from the rest of equations. The following argument rules out also the positive root $\xi_{1}=\frac{1}{2}(3+\sqrt{13})$ of the polynomial $\xi_{1}^{2}-3 \xi_{1}-1$. The remaining polynomials of the Gröbner basis determine the corresponding $\xi_{0}=$ $\frac{1}{2}(3+\sqrt{13})$. But the leading term of the denominator (2.6) expansion

$$
D\left(\xi_{0} h, \xi_{1} h\right)=\kappa_{0}^{4}\left(\xi_{0}^{2} \xi_{1}^{2}-9 \xi_{0} \xi_{1}-3 \xi_{1}-3 \xi_{0}-1\right) h^{4}+\mathcal{O}\left(h^{5}\right)
$$

vanishes at

$$
\xi_{0}=\xi_{1}=\frac{1}{2}(3+\sqrt{13}) .
$$

However, the expansion of the second rational equation in (2.7) at the values (3.16) is

$$
e_{4}\left(\xi_{0} h, \xi_{1} h, \mu_{0}\left(\xi_{0} h, \xi_{1} h\right), \mu_{1}\left(\xi_{0} h, \xi_{1} h\right)=\frac{135}{2}(659-183 \sqrt{13}) \kappa_{0}^{2} h^{3}+\mathcal{O}\left(h^{4}\right) .\right.
$$

So the solution (3.16) is extraneous, and it is due to the switch from the rational (2.7) to the polynomial form (2.8) of the equations. This yields the possible choices $\xi_{1}=1,3, u_{0}, u_{1}$, where $u_{0} \approx 1.27927$ and $u_{1} \approx 2.55324$ are positive roots of $\pi_{5}$. The rest of the Gröbner basis gives the corresponding values $\xi_{0}=1,3, u_{1}, u_{0}$ respectively. The last three pairs $\left(\xi_{0}, \xi_{1}\right)$ produce also a nonsingular Jacobian of the polynomial part of the scaled equations

$$
\begin{aligned}
& \frac{1}{h^{7}} e_{2}\left(\xi_{0} h, \xi_{1} h, \mu_{0}\left(\xi_{0} h, \xi_{1} h\right), \mu_{1}\left(\xi_{0} h, \xi_{1} h\right)\right)+\mathcal{O}(h), \\
& \frac{1}{h^{12}} e_{4}\left(\xi_{0} h, \xi_{1} h, \mu_{0}\left(\xi_{0} h, \xi_{1} h\right), \mu_{1}\left(\xi_{0} h, \xi_{1} h\right)\right)+\mathcal{O}(h),
\end{aligned}
$$

at the limit solution. So one can follow the steps of the proof of Lemma 3.3 and confirm the following lemma in a similar manner. Of course, some further expansions have to be carried out on the way, but Lemma 3.1 shortens them significantly: if $\lambda_{0}=\xi_{0} h+\zeta_{0} h^{2}+$ $\mathcal{O}\left(h^{3}\right), \lambda_{1}=\xi_{1} h+\zeta_{1} h^{2}+\mathcal{O}\left(h^{3}\right)$ is an admissible solution pair then if $\xi_{0} \neq \xi_{1}$, there exists also an admissible solution pair $\lambda_{0}=\xi_{1} h-\zeta_{1} h^{2}+\mathcal{O}\left(h^{3}\right), \lambda_{0}=\xi_{0} h-\zeta_{1} h^{2}+\mathcal{O}\left(h^{3}\right)$. If $\xi_{0}=\xi_{1}$ and the solution is simple, then it should be of the form

$$
\lambda_{0}=\xi_{0} h+\zeta_{0} h^{2}+\mathcal{O}\left(h^{3}\right), \quad \lambda_{1}=\xi_{0} h-\zeta_{0} h^{2}+\mathcal{O}\left(h^{3}\right) .
$$

Lemma 3.4. Let the assumptions of Lemma 3.2 be fulfilled. The system (2.4) has in the case $\alpha=\beta=1$ three simple asymptotic solutions given by

$$
\begin{aligned}
& \lambda_{0}=\lambda_{0}(h):=3 h+\frac{11 \kappa_{1}}{12 \kappa_{0}^{2}} h^{2}+\mathcal{O}\left(h^{3}\right), \quad \lambda_{1}=-\lambda_{0}(-h), \\
& \mu_{0}=\mu_{0}(h):=-12 h+\mathcal{O}\left(h^{2}\right), \quad \mu_{1}=\mu_{0}(-h),
\end{aligned}
$$

and

$$
\begin{aligned}
& \lambda_{0}=\lambda_{0}(h):=u_{0} h+\zeta_{0} h^{2}+\mathcal{O}\left(h^{3}\right), \quad \lambda_{1}=\lambda_{1}(h):=u_{1} h+\zeta_{1} h^{2}+\mathcal{O}\left(h^{3}\right), \\
& \mu_{0}=\mu_{0}(h):=\nu_{0} h+\mathcal{O}\left(h^{2}\right), \quad \mu_{1}=\mu_{1}(h):=\nu_{1} h+\mathcal{O}\left(h^{2}\right), \\
& \lambda_{0}=-\lambda_{1}(-h), \quad \lambda_{1}=-\lambda_{0}(-h), \quad \mu_{0}=\mu_{1}(-h), \quad \mu_{1}=\mu_{0}(-h),
\end{aligned}
$$


with

$$
\begin{aligned}
& u_{0} \approx 1.27927, \quad u_{1} \approx 2.55324, \quad \zeta_{0}=\frac{0.653557 \kappa_{1}}{\kappa_{0}}, \quad \zeta_{1}=-\frac{0.28438 \kappa_{1}}{\kappa_{0}}, \\
& \nu_{0}=-1.79886, \quad \nu_{1}=11.0729 .
\end{aligned}
$$

The case $\xi_{0}=\xi_{1}=1$ requires some additional attention since at this limit solution the Jacobian of Eq. (3.17) vanishes identically as $h \rightarrow 0$.

Lemma 3.5. Let us suppose again that the assumptions of Lemma 3.2 are fulfilled. Besides the asymptotic solutions established in Lemma 3.4, the system (2.4) has for $\alpha=\beta=1$ in the general case an odd number $\leq 15$ of simple asymptotic solutions. All of them are of the form

$$
\begin{array}{ll}
\lambda_{0}=\lambda_{0}(h)=h+\zeta h^{2}+\varrho h^{3}+\theta h^{4}+\mathcal{O}\left(h^{5}\right), & \lambda_{1}=-\lambda_{0}(-h), \\
\mu_{0}=\mu_{0}(h)=-2 \zeta h^{2}-6 \varrho h^{3}+\chi h^{4}+\mathcal{O}\left(h^{5}\right), & \mu_{1}=\mu_{0}(-h) .
\end{array}
$$

Here, $\zeta$ and $\rho$ are real solutions of a particular system of polynomial equations (3.22). The term general excludes some exceptional relations between the coefficients of the curvature expansions which imply a zero of multiplicity at least 3 of the polynomial (3.25).

Proof. Since the expansion of the unknowns $\lambda_{i}$ starts with the linear term $h$, the expansion should be of the form

$$
\lambda_{0}=h+h\left(\zeta_{0} h^{\alpha}+\mathcal{o}\left(h^{\alpha}\right)\right), \lambda_{1}=h+h\left(\zeta_{1} h^{\beta}+\mathcal{o}\left(h^{\beta}\right)\right), \quad \zeta_{0} \neq 0, \quad \zeta_{1} \neq 0,
$$

with $\alpha, \beta>0$. The leading terms

$$
\begin{aligned}
& e_{2}=15 \kappa_{0}^{6} \zeta_{0}^{3} h^{3 \alpha+7}+\mathcal{o}\left(h^{3 \alpha+7}\right), \quad 0<\alpha<1 \wedge \alpha<\beta, \\
& e_{2}=-15 \kappa_{0}^{6} \zeta_{1}^{3} h^{3 \beta+7}+\mathcal{o}\left(h^{3 \beta+7}\right), \quad 0<\beta<1 \wedge \beta<\alpha,
\end{aligned}
$$

rule out all the possible exponents in $\mathcal{D}=\{(\alpha, \beta) \mid 0<\alpha<1 \vee 0<\beta<1\}$ but $\alpha=\beta$. In the latter case we obtain

$$
\begin{aligned}
& e_{2}=15 \kappa_{0}^{6}\left(\zeta_{0}-\zeta_{1}\right)\left(\zeta_{0}+\zeta_{1}\right)^{2} h^{3 \alpha+7}+\mathcal{o}\left(h^{3 \alpha+7}\right), \\
& e_{4}=1800 \kappa_{0}^{10}\left(\zeta_{0}+\zeta_{1}\right)^{2}\left(4 \zeta_{0}+\zeta_{1}\right) h^{3 \alpha+12}+\mathcal{o}\left(h^{3 \alpha+12}\right) .
\end{aligned}
$$

This additionally implies $\zeta_{1}=-\zeta_{0}$, but then

$$
e_{2}=-20 \kappa_{0}^{6} \zeta_{0}^{5} h^{5 \alpha+7}+\mathcal{o}\left(h^{5 \alpha+7}\right)
$$

rules out $\mathcal{D}$ completely. So we may assume $\alpha=\beta=1$, but we additionally allow that $\zeta_{0}, \zeta_{1}$ might depend on $h$ as decreasing functions of the argument. Thus we obtain

$$
\begin{aligned}
& e_{2}=30 \kappa_{0}^{5} \Delta_{0}^{2}\left(2 \kappa_{0} \Delta_{0}-\kappa_{1}\right) h^{10}+\cdots \\
& e_{4}=1800 \kappa_{0}^{9} \Delta_{0}^{2}\left(20 \kappa_{0} \Delta_{0}+12 \kappa_{0} \Delta_{1}-3 \kappa_{1}\right) h^{15}+\cdots,
\end{aligned}
$$


with $\Delta_{0}:=\frac{\zeta_{0}+\zeta_{1}}{2}, \Delta_{1}:=\frac{\zeta_{0}-\zeta_{1}}{2}$. A particular linear combination of these equations reveals

$$
\begin{gathered}
\frac{1}{36000 \kappa_{0}^{10} h^{15}}\left(e_{4}-180 \kappa_{0}^{4} h^{5} e_{2}\right)=\pi_{6}\left(\Delta_{0}, \Delta_{1}\right)+\mathcal{O}\left(h^{4}\right), \\
\pi_{6}(u, v):=u^{3}+\pi_{7}(u, v) u^{2} h+\pi_{8}(u, v) u h^{2}+\pi_{9}(u, v) h^{3} .
\end{gathered}
$$

The coefficients of polynomials $\pi_{i}, i=7,8,9$, depend on the data curvature only, but not on $h$. The expansion (3.21) clearly implies

$$
\Delta_{0}=\rho h+\mathcal{O}(h), \quad \rho \in \mathbb{R} .
$$

Let also $\Delta_{1}=\zeta+\mathcal{o}(1), \zeta \in \mathbb{R}$. These assumptions give two polynomial equations for the unknowns $\rho$ and $\zeta$. From (3.20) we obtain

$$
\frac{1}{h^{2}} e_{2}=: \pi_{10}(\zeta, \rho)+\mathcal{O}(1), \quad \pi_{10}(\zeta, \rho)=\pi_{12}(\zeta) \rho^{2}+\pi_{13}(\zeta) \rho+\pi_{14}(\zeta),
$$

and the relation (3.21) gives

$$
\begin{aligned}
& \frac{1}{h^{3}} \pi_{6}(\rho h+\mathcal{o}(h), \zeta+\mathcal{o}(1))=: \pi_{11}(\zeta, \rho)+\mathcal{o}(1), \\
& \pi_{11}(\zeta, \rho)=\rho^{3}+\pi_{15}(\zeta) \rho^{2}+\pi_{16}(\zeta) \rho+\pi_{17}(\zeta) .
\end{aligned}
$$

The coefficient polynomials involved in $\pi_{10}$ and $\pi_{11}$ are

$$
\begin{aligned}
& \pi_{12}(\zeta)=4 \kappa_{0} \zeta-\kappa_{1}, \quad \pi_{13}(\zeta)=-\frac{2}{3} \kappa_{0} \zeta^{3}-\frac{3}{2} \kappa_{1} \zeta^{2}+\cdots \\
& \pi_{14}(\zeta)=-\frac{2}{3} \kappa_{0} \zeta^{5}+\kappa_{1} \zeta^{4}+\cdots, \\
& \pi_{15}(\zeta)=-\frac{7}{10} \zeta^{2}-\frac{9 \kappa_{1}}{20 \kappa_{0}} \zeta+\cdots, \quad \pi_{16}(\zeta)=-\frac{13}{60} \zeta^{4}+\frac{23 \kappa_{1}}{40 \kappa_{0}} \zeta^{3}+\cdots \\
& \pi_{17}(\zeta)=-\frac{43}{360} \zeta^{6}+\frac{29 \kappa_{1}}{120 \kappa_{0}} \zeta^{5}+\cdots
\end{aligned}
$$

The system of polynomial equations that should determine $\zeta$ and $\rho$ finalizes as

$$
\pi_{10}(\zeta, \rho)=0, \quad \pi_{11}(\zeta, \rho)=0
$$

A little of the computer algebra work verifies a reduction

$$
\pi_{12}^{2}(\zeta) \pi_{11}(\zeta, \rho)=\pi_{18}(\zeta, \rho) \pi_{10}(\zeta, \rho)+\pi_{19}(\zeta) \rho+\pi_{20}(\zeta)
$$

If the unknowns $\zeta, \rho$ satisfy (3.22), $\rho$ could be expressed in terms of $\zeta$ from (3.23),

$$
\rho=-\frac{\pi_{20}(\zeta)}{\pi_{19}(\zeta)}
$$


But then the polynomial form of the first equation in (3.22) factorizes as

$$
\pi_{19}^{2}(\zeta) \pi_{10}\left(\zeta,-\pi_{20}(\zeta) / \pi_{19}(\zeta)\right)=\pi_{12}^{2}(\zeta) \pi_{21}(\zeta)
$$

where

$$
\pi_{21}(\zeta)=\frac{700}{243} \kappa_{0}^{3} \zeta^{15}-\frac{875}{81} \kappa_{0}^{2} \kappa_{1} \zeta^{14}+\cdots
$$

Thus Eq. (3.25) has an odd number of simple real solutions in general, and from (3.24) consequently the system (3.22) too. The exceptional cases are excluded by the assumption

$$
\operatorname{gcd}\left(\pi_{21}, \pi_{21}^{\prime}, \pi_{21}^{\prime \prime}\right)=\text { const. }
$$

If $(\zeta, \rho)$ is a simple solution of the system (3.22), the unknowns $\Delta_{i}$ admit series expansion in $h$, and so do $\lambda_{i}$. By (3.18), the latter has to be of the form (3.19). The expansion of $\mu_{i}$ is then straightforward. The proof is concluded.

Theorem 3.1. Suppose that $\kappa_{0} \neq 0$, and $h>0$ is small enough. The system (2.4) has asymptotically an $\mathcal{O}(1)$ solution, determined in Lemma 3.3. Further, all the solutions, established in Lemma 3.4, are of the order $\mathcal{O}\left(h^{2}\right)$. At last, there is an odd number of optimal $\mathcal{O}\left(h^{6}\right)$ solutions, confirmed in Lemma 3.5.

Proof. The existence part of the assertions has already been proved in Lemmas $3.3,3.4$ and 3.5. It remains to prove the asymptotic approximation order only. Let us recall the parametric distance (see, e.g., [18]) as a measure of the distance between parametric curves $\boldsymbol{f}:[c, d] \rightarrow \mathbb{R}^{d}$ and $\boldsymbol{g}:[a, b] \rightarrow \mathbb{R}^{d}$, defined as

$$
\operatorname{dist}_{P}(\boldsymbol{f}, \boldsymbol{g}):=\inf _{\varphi} \max _{a \leq t \leq b}\|(\boldsymbol{f} \circ \varphi)(t)-\boldsymbol{g}(t)\|,
$$

where the infimum is taken among all diffeomorphisms $\varphi:[a, b] \rightarrow[c, d]$, and $\|\cdot\|$ is the usual Euclidean norm. Any particular reparameterization $\varphi$ gives an upper bound on the parametric distance (3.26). Here, $\boldsymbol{f}=\boldsymbol{r}:\left[-\frac{h}{2}, \frac{h}{2}\right] \rightarrow \mathbb{R}^{d}$ is a smooth data curve, and $\boldsymbol{g}=\boldsymbol{p}:[0,1] \rightarrow \mathbb{R}^{d}$ is the quintic PH interpolating polynomial curve.

Let us consider the asymptotic solutions, determined in Lemma 3.5, and let us choose the reparameterization $\varphi:[0,1] \rightarrow\left[-\frac{h}{2}, \frac{h}{2}\right]$ as a quintic polynomial, determined by conditions

$$
\varphi(0)=-\frac{h}{2}, \quad \varphi(1)=\frac{h}{2}, \quad \varphi^{\prime}(0)=\lambda_{0}, \quad \varphi^{\prime}(1)=\lambda_{1}, \quad \varphi^{\prime \prime}(0)=\mu_{0}, \quad \varphi^{\prime \prime}(1)=\mu_{1} .
$$

The quintic polynomial curve $\boldsymbol{p}$ agrees with $\boldsymbol{r} \circ \varphi$ three-fold at 0 and at 1 , respectively. Thus the Newton form of the interpolation error reads

$$
\begin{aligned}
(\boldsymbol{r} \circ \varphi)(t)-\boldsymbol{p}(t) & =t^{3}(t-1)^{3}[0,0,0,1,1,1, t](\boldsymbol{r} \circ \varphi) \\
& =t^{3}(t-1)^{3}(\boldsymbol{r} \circ \varphi)^{(6)}\left(\eta_{t}\right), \quad \eta_{t} \in[0,1] .
\end{aligned}
$$


The chain rule applied to $r \circ \varphi$ reveals that the error term would be $\mathcal{O}\left(h^{6}\right)$ if the derivatives of the reparameterization satisfy $\varphi^{(\ell)}=\mathcal{O}\left(h^{\ell}\right)$ for $\ell=1,2, \cdots, 5$ on $[0,1]$. Note additionally that $\varphi^{(6)} \equiv 0$. The simplest way to confirm the assertion is to compute the divided difference table that determines Newton forms of $\varphi$. The upper diagonal that gives the first one reads

$$
\begin{aligned}
& {[0] \varphi=-\frac{h}{2}} \\
& {[0,0] \varphi=\lambda_{0}=h+\mathcal{O}\left(h^{2}\right)} \\
& {[0,0,0] \varphi=\frac{\mu_{0}}{2}=-\zeta h^{2}+\mathcal{O}\left(h^{3}\right),} \\
& {[0,0,0,1] \varphi=h-\lambda_{0}-\frac{\mu_{0}}{2}=2 \rho h^{3}+\mathcal{O}\left(h^{4}\right),} \\
& {[0,0,0,1,1] \varphi=-3 h+2 \lambda_{0}+\lambda_{1}+\frac{\mu_{0}}{2}=\left(\theta+\frac{1}{2} \chi\right) h^{4}+\mathcal{O}\left(h^{5}\right),} \\
& {[0,0,0,1,1,1] \varphi=6 h-3 \lambda_{0}-3 \lambda_{1}-\frac{\mu_{0}}{2}+\frac{\mu_{1}}{2}=\mathcal{O}\left(h^{5}\right) .}
\end{aligned}
$$

So

$$
\varphi^{\prime}(t)=\left([0] \varphi+[0,0] \varphi t+[0,0,0] \varphi t^{2}+\cdots\right)^{\prime}=h+\mathcal{O}\left(h^{2}\right),
$$

and $\varphi$ is a regular reparameterization that satisfies $\varphi^{(\ell)}=\mathcal{O}\left(h^{\ell}\right), \ell=2,3, \cdots, 5$. This concludes the proof for the $\mathcal{O}\left(h^{6}\right)$ solutions. The $\mathcal{O}(1)$ case of Lemma 3.3 follows from the fact that the interpolating curve is bounded independently of $h$. For the $\mathcal{O}\left(h^{2}\right)$ case one can choose a parabola that interpolates the values $-\frac{h}{2}, \lambda_{0}, \frac{h}{2}$ at $0,0,1$ as a reparameterization $\varphi$. The proof is completed.

Quite clearly, if the interpolated curve is a quintic PH curve one of the $\mathcal{O}\left(h^{6}\right)$ solutions would reproduce it exactly and the others, if they exist, would be $\mathcal{O}\left(h^{6}\right)$ and not better. On the other hand, the solutions of Lemmas 3.3 and 3.4 could not do better than $\mathcal{O}(1)$ and $\mathcal{O}\left(h^{2}\right)$, respectively, in general. A constant

$$
\Theta(\boldsymbol{p}):=\min _{-h / 2 \leq s \leq h / 2} \Theta(\boldsymbol{p} ; s), \quad \Theta(\boldsymbol{p} ; s):=\left\|\boldsymbol{r}(s)-\boldsymbol{p}\left(\frac{1}{2}\right)\right\|,
$$

bounds the Hausdorff distance between the curve $\boldsymbol{r}$ and the PH interpolant $\boldsymbol{p}$ from below. The solution from Lemma 3.3 yields

$$
\Theta(\boldsymbol{p})=\frac{5}{4 \tau_{0}}+\mathcal{O}(h) .
$$

For the solutions of Lemma 3.4 we obtain

$$
\Theta(\boldsymbol{p} ; s)^{2}=h^{2} \pi_{22}^{2}\left(\frac{s}{h}\right)+\mathcal{O}\left(h^{3}\right),
$$

where $\pi_{22}$ is a linear polynomial with coefficients independent of $h$. But $\Theta(\boldsymbol{p})^{2}=$ $\mathcal{O}\left(h^{4}\right)$, so the first term of $s / h$ should be a root of $\pi_{22}$. This gives the values $s$ that 
minimize the right-hand side of (3.27) for the three solutions as

$$
s=\mathcal{O}\left(h^{2}\right), \quad s \approx \pm 0.343966 h+\mathcal{O}\left(h^{2}\right),
$$

and the lower bounds

$$
\Theta(\boldsymbol{p})=\frac{\sqrt{\kappa_{0}^{4}+\kappa_{1}^{2}}}{8 \kappa_{0}} h^{2}+\mathcal{O}\left(h^{3}\right), \quad \Theta(\boldsymbol{p}) \approx \frac{\sqrt{0.00311317 \kappa_{0}^{4}+0.112154 \kappa_{1}^{2}}}{10 \kappa_{0}} h^{2}+\mathcal{O}\left(h^{3}\right)
$$

respectively. Theorem 3.1, and the previous discussion prove that the Hausdorff distance dist $_{H}(\boldsymbol{r}, \boldsymbol{p})$ behaves precisely like

$$
\operatorname{dist}_{H}(\boldsymbol{r}, \boldsymbol{p})=\text { const }+\mathcal{O}(h), \text { const }>0, \quad \operatorname{dist}_{H}(\boldsymbol{r}, \boldsymbol{p})=\text { const } h^{2}+\mathcal{O}\left(h^{3}\right), \text { const }>0,
$$

for the solutions of Lemmas 3.3 and 3.4 respectively. So, for $h$ small enough, they should differ significantly from the optimal order solutions of Lemma 3.5. This explains the background of the numerical algorithm developed in the next section.

\section{Algorithm and numerical examples}

In practical applications there is usually a large number of data to be interpolated. The presented $G^{2} \mathrm{PH}$ quintic interpolation scheme constructs polynomial curves which can be joined together to the spline entirely locally. Asymptotic analysis reveals that there might exist many solutions of the interpolation problem considered and one must decide which of them is to be used on each spline segment. Quite clearly, the solution with the best approximation order, i.e., order six in this case, should be chosen. But this can be done only in an asymptotic way, i.e., when the data is taken from a smooth parametric curve over some small parameter interval. To avoid this limitation we propose an algorithm based on a continuation method that can be used also for the nonasymptotic data.

The idea is to choose a particular set of data for which all the solutions can be computed and distinguished by the order of approximation. Then this particular data is connected with the original data by a smooth homotopy. Starting with the "best solution" of the particular interpolation problem, we trace this solution by a continuation method to reach the original data. In this way the asymptotic results are carried over to the nonasymptotic data.

Particular data

$$
V^{*}:=\left(\boldsymbol{f}_{0}^{*}, \quad \boldsymbol{d}_{0}^{*}, \quad \Delta \boldsymbol{P}_{0}^{*}, \quad \boldsymbol{d}_{1}^{*}, \quad \boldsymbol{f}_{1}^{*}\right)
$$

are taken from a quintic PH curve

$$
\boldsymbol{p}^{*}(t):=\left(\begin{array}{c}
\frac{1}{48}\left(-48 t^{4}+184 t^{2}-43\right)+\frac{536}{625} \\
\frac{1}{72}\left(48 t^{5}-280 t^{3}+435 t+184\right)-\frac{54992}{28125} \\
\frac{1}{6}\left(8 t^{4}-14 t^{2}+3\right)-\frac{298}{625}
\end{array}\right)
$$


Table 3: The Hausdorff distance and parameters $\lambda_{i}, \mu_{i}$ for seven solutions of the particular case, where $h$ is the length of the curve $\boldsymbol{p}^{*}$ on the interval $\left[t_{0}, t_{1}\right]$.

\begin{tabular}{||c|c|c|c|c|c||}
\hline & Hausdorff distance & $\lambda_{0}$ & $\lambda_{1}$ & $\mu_{0}$ & $\mu_{1}$ \\
\hline 1 & 0 & $0.994512 h$ & $0.994512 h$ & $0.0271798 h^{2}$ & $-0.0271798 h^{2}$ \\
2 & $1.1143 \times 10^{-5}$ & $0.812225 h$ & $1.20396 h$ & $0.248020 h^{2}$ & $0.335774 h^{2}$ \\
3 & $1.1143 \times 10^{-5}$ & $1.20396 h$ & $0.812225 h$ & $-0.335774 h^{2}$ & $-0.248020 h^{2}$ \\
4 & $3.4566 \times 10^{-3}$ & $1.22545 h$ & $2.52048 h$ & $-10.9048 h$ & $1.66426 h$ \\
5 & $3.4566 \times 10^{-3}$ & $2.52048 h$ & $1.22545 h$ & $-1.66426 h$ & $10.9048 h$ \\
6 & $4.7791 \times 10^{-2}$ & $3.03129 h$ & $3.03129 h$ & $-12.3336 h$ & $12.3336 h$ \\
7 & 4.2009 & 24.8279 & 24.8279 & -110.916 & 110.916 \\
\hline
\end{tabular}

at the parameter values $-\frac{1}{10}$ and $\frac{1}{10}$. For the numerical part it is convenient to separate geometric properties of the data Gram matrix in the distance and the angle part by

$$
\delta_{i}:=+\sqrt{g_{i i}}, \quad i=0,1, \cdots, 4, \quad c_{i j}:=\frac{1}{\delta_{i} \delta_{j}} g_{i j}, \quad i, j=0,1, \cdots, 4 .
$$

The constants (4.3) that correspond to the particular data (4.1) will be denoted by $\delta_{i}^{*}, c_{i j}^{*}$. Clearly, one of the solutions is the curve $\boldsymbol{p}^{*}$ itself, but there are six additional ones. The unknown parameters for all of them are given in Table 3. Solutions 2 and 3 are order 6 approximants of $\boldsymbol{p}^{*}$, solutions 4,5 and 6 have order two and the last solution is far away from $\boldsymbol{p}^{*}$. The results in Table 3 match the results from Lemma 3.3, Lemma 3.4 and Lemma 3.5. One can see that parameters $\lambda_{i}$ and $\mu_{i}, i=0,1$, for order six approximants differ significantly from parameters for the other solutions. The same can be observed already from Table 1 .

The homotopy algorithm has three steps:

1. Translation, rotation and scaling of the original data.

2. Construction of a homotopy function.

3. Solving the system by a continuation method.

Before we explain each step in detail, let us shortly recall the theory of spatial rotations. As it is known, they are closely connected with the quaternions. Namely, any nonzero quaternion $\boldsymbol{q}= \pm\left[q_{0},\left(q_{1}, q_{2}, q_{3}\right)^{T}\right] \in \mathbb{H}$, where $\mathbb{H}$ is a field of quaternions, defines a rotation $R=\operatorname{Rot}(\boldsymbol{q})$, given by

$$
\operatorname{Rot}(\boldsymbol{q})=\frac{1}{\|\boldsymbol{q}\|^{2}}\left(\begin{array}{ccc}
q_{0}^{2}+q_{1}^{2}-q_{2}^{2}-q_{3}^{2} & 2\left(q_{1} q_{2}-q_{0} q_{3}\right) & 2\left(q_{1} q_{3}+q_{0} q_{2}\right) \\
2\left(q_{1} q_{2}+q_{0} q_{3}\right) & q_{0}^{2}-q_{1}^{2}+q_{2}^{2}-q_{3}^{2} & 2\left(q_{2} q_{3}-q_{0} q_{1}\right) \\
2\left(q_{1} q_{3}-q_{0} q_{2}\right) & 2\left(q_{2} q_{3}+q_{0} q_{1}\right) & q_{0}^{2}-q_{1}^{2}-q_{2}^{2}+q_{3}^{2}
\end{array}\right) .
$$

The mapping Rot $: \mathbb{H} \rightarrow\left\{R \in \mathbb{R}^{3 \times 3}, R^{T} R=1\right.$, $\left.\operatorname{det} R=1\right\}$ is called a kinematical mapping. Conversely, every rotation can be represented by two antipodal unit quaternions $\pm \boldsymbol{q} \in \mathbb{H},\|\boldsymbol{q}\|=1$ (see [6]). 
STEP 1:

The original data $V=\left(\boldsymbol{f}_{0}, \boldsymbol{d}_{0}, \Delta \boldsymbol{P}_{0}, \boldsymbol{d}_{1}, \boldsymbol{f}_{1}\right)$ are translated, rotated and scaled as

$$
\begin{aligned}
\boldsymbol{P}_{0} & \rightarrow(0,0,0)^{T}, \\
\boldsymbol{P}_{1} & \rightarrow \frac{\delta_{2}^{*}}{\delta_{2}} R \Delta \boldsymbol{P}_{0}, \\
\boldsymbol{d}_{i} & \rightarrow R \boldsymbol{d}_{i}, \quad i=0,1, \\
\boldsymbol{f}_{i} & \rightarrow \frac{\delta_{2}}{\delta_{2}^{*}} R \Delta \boldsymbol{f}_{i}, \quad i=0,1,
\end{aligned}
$$

where $R \in \mathbb{R}^{3 \times 3}$ is a rotation matrix that transforms a vector $\Delta \boldsymbol{P}_{0}$ onto $\frac{\delta_{2}}{\delta_{2}^{*}} \Delta \boldsymbol{P}^{*}$. More precisely,

$$
R=\operatorname{Rot}\left(\left[\cos \frac{\phi}{2}, \sin \frac{\phi}{2} \boldsymbol{a}\right]\right),
$$

where the rotation axis $\boldsymbol{a}$ and the rotation angle $\phi$ are given as

$$
\boldsymbol{a}=\frac{1}{\delta_{2} \delta_{2}^{*}} \Delta \boldsymbol{P}_{0} \times \Delta \boldsymbol{P}_{0}^{*}, \quad \cos \phi=\frac{1}{\delta_{2} \delta_{2}^{*}} \Delta \boldsymbol{P}_{0} \cdot \Delta \boldsymbol{P}_{0}^{*} .
$$

Note that the solution of the transformed problem (4.4) is just a scaled solution of the original one

$$
\lambda_{i} \rightarrow \frac{\delta_{2}^{*}}{\delta_{2}} \lambda_{i}, \quad \mu_{i} \rightarrow \frac{\delta_{2}^{*}}{\delta_{2}} \mu_{i}, \quad i=0,1 .
$$

STEP 2: Construction of a homotopy function that connects the particular data $V^{*}$ with the transformed data (4.4).

Since $\boldsymbol{d}_{i} \perp \boldsymbol{f}_{i}$ and $\boldsymbol{d}_{i}^{*} \perp \boldsymbol{f}_{i}^{*}, i=0,1$,

$$
Q_{i}:=\left(\boldsymbol{d}_{i}, \quad \frac{1}{\delta_{4 i}} \boldsymbol{f}_{i}, \quad \frac{1}{\delta_{4 i}} \boldsymbol{d}_{i} \times \boldsymbol{f}_{i}\right) \quad \text { and } \quad Q_{i}^{*}:=\left(\boldsymbol{d}_{i}^{*}, \quad \frac{1}{\delta_{4 i}^{*}} \boldsymbol{f}_{i}^{*}, \quad \frac{1}{\delta_{4 i}^{*}} \boldsymbol{d}_{i}^{*} \times \boldsymbol{f}_{i}^{*}\right)
$$

are the rotation matrices. Let $\boldsymbol{q}_{i}$ and $\boldsymbol{q}_{i}^{*}$ be the unit quaternions, that correspond to $Q_{i}$ and $Q_{i}^{*}$. The sign must be chosen in such a way that $\boldsymbol{q}_{i}^{T} \boldsymbol{q}_{i}^{*}>0, i=0,1$. The quaternion line

$$
\boldsymbol{h}_{i}(t):=(1-t) \boldsymbol{q}_{i}^{*}+t \boldsymbol{q}_{i}, \quad i=0,1,
$$

defines a rational spherical motion of degree two. The homotopy function is defined as

$$
H:[0,1] \rightarrow V, \quad H(t)=\left(f_{0}(t), \quad d_{0}(t), \quad \Delta \boldsymbol{P}_{0}(t), \quad \boldsymbol{d}_{1}(t), \quad \boldsymbol{f}_{1}(t)\right),
$$

where

$$
\begin{aligned}
& \Delta \boldsymbol{P}_{0}(t):=\Delta \boldsymbol{P}_{0} \\
& \boldsymbol{f}_{i}(t):=\left((1-t) \delta_{4 i}^{*}+t \delta_{4 i}\right) \operatorname{Rot}\left(\boldsymbol{h}_{i}(t)\right)(0, \quad 1, \quad 0)^{T}, \quad i=0,1, \\
& \boldsymbol{d}_{i}(t):=\operatorname{Rot}\left(\boldsymbol{h}_{i}(t)\right)\left(\begin{array}{lll}
1, & 0, & 0
\end{array}\right)^{T}, \quad i=0,1 .
\end{aligned}
$$


Quite clearly, $H(0)=V^{*}$ and $H(1)=V$, where $V$ denotes the data after the first step.

STEP 3: Continuation method.

Choose one solution from Table 3. Trace this solution by a continuation method along the homotopy path defined by $H$. The result is the solution of the interpolation problem for data (4.4).

The proposed algorithm works efficiently for most data configurations, however the following problems may occur. The solution that we trace can be lost along the homotopy path if two solutions meet and turn into a pair of complex solutions or if the solution crosses the boundary of the domain

$$
\left\{\left(\lambda_{0}, \lambda_{1}, \mu_{0}, \mu_{1}\right): \lambda_{i}>0, \mu_{i} \neq 0, i=0,1\right\} .
$$

The reason for this problem is usually that the interpolation problem does not have a solution at all. It may happen that the solution exists, but the homotopy path contains parts where it does not. In this case we should use the extended continuation method that enables us to trace the solutions in the complex domain and return back.

As an example, let us connect particular data $V^{*}=\left(\boldsymbol{f}_{0}^{*}, \boldsymbol{d}_{0}^{*}, \Delta \boldsymbol{P}_{0}^{*}, \boldsymbol{d}_{1}^{*}, \boldsymbol{f}_{1}^{*}\right)$, taken from the quintic PH curve (4.2) at parameters $-\frac{1}{10}$ and $\frac{1}{10}$, with the data $V=\left(\boldsymbol{f}_{0}, \boldsymbol{d}_{0}\right.$, $\left.\Delta \boldsymbol{P}_{0}, \boldsymbol{d}_{1}, \boldsymbol{f}_{1}\right)$, taken from the curve (3.1) at parameters 0 and $\frac{1}{4}$. Let us trace the first solution from Table 3 by using homotopy approach considered above. In the first step of the algorithm, the original data $V$ are transformed to data $\widetilde{V}=\left(\widetilde{\boldsymbol{f}}_{0}, \widetilde{\boldsymbol{d}}_{0}, \widetilde{\Delta \boldsymbol{P}_{0}}, \widetilde{\boldsymbol{d}}_{1}, \widetilde{\boldsymbol{f}}_{1}\right)$, as described in (4.4) (see Fig. 5). Secondly, homotopy path that connects the transformed data to the particular data $V^{*}$ has to be constructed as presented in (4.5) (see

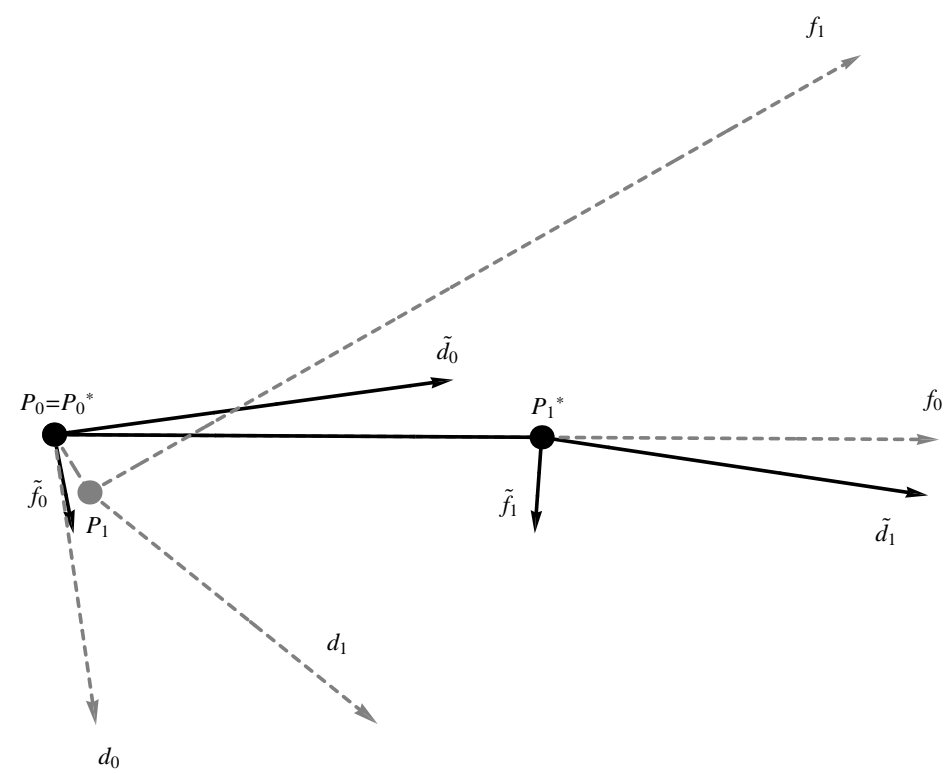

Figure 5: Original data $V$ (dashed gray) and the data $\widetilde{V}$ after the first step of the algorithm (black). 


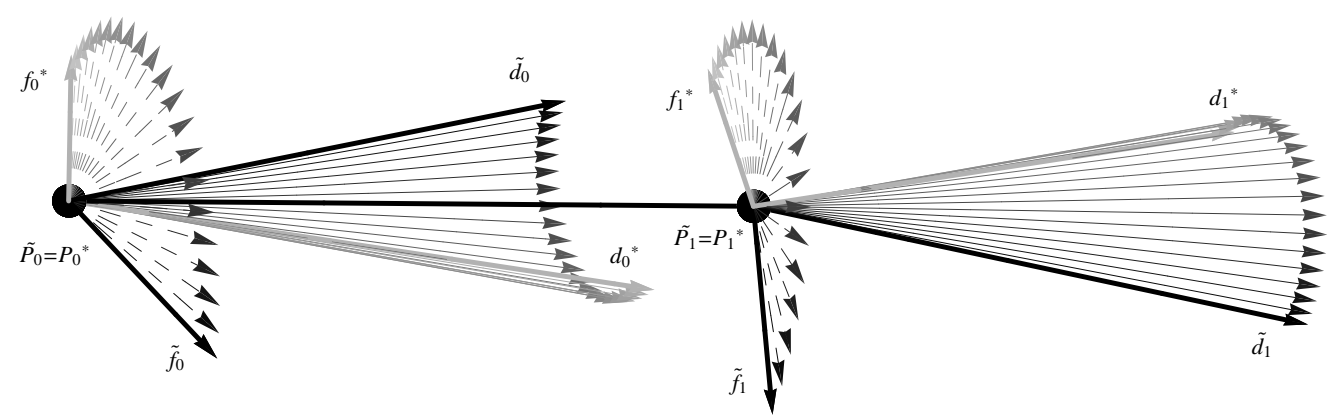

Figure 6: Homotopy from the particular data $V^{*}$ (gray) to the transformed original data $\widetilde{V}$ (black).
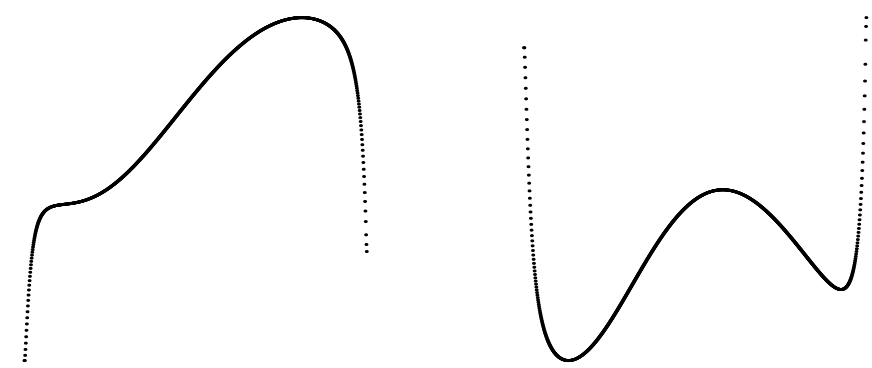

Figure 7: Transformations of parameters $\lambda_{0}$ (left) and $\lambda_{1}$ (right) along the homotopy path from the best solution of the transformed original data $\widetilde{V}$ to the best solution for the particular data $V^{*}$.

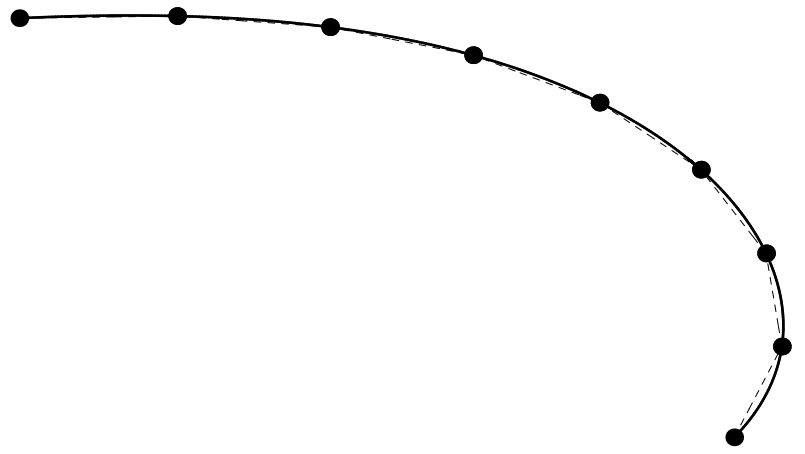

Figure 8: A $G^{2}$ quintic $\mathrm{PH}$ spline interpolating the curve (3.1) at parameters $\frac{i}{4}, i=0,1, \cdots, 8$.

Fig. 6). Finally, the selected solution is traced by the continuation method along the obtained homotopy path. As expected, parameters $\lambda_{i}$ and $\mu_{i}, i=0,1$, obtained from the first row in Table 3, after transformation in the first step of the algorithm, transform to the parameters given in the first row in Table 1 (see Fig. 7). As the second example, let us consider an interpolation by a $G^{2}$ quintic $\mathrm{PH}$ spline. Let the data be taken from the curve (3.1) at parameters $\frac{i}{4}, i=0,1, \cdots, 8$. Using the same approach as in the previous example for each particular segment we obtain the final $G^{2} \mathrm{PH}$ spline shown in Fig. 8. 


\section{References}

[1] Gudrun Albrecht and Rida T. Farouki, Construction of $C^{2}$ Pythagorean-hodograph interpolating splines by the homotopy method, Adv. Comput. Math. 5 (1996), no. 4, 417-442.

[2] Eugene L. Allgower and Kurt Georg, Numerical continuation methods, Springer Series in Computational Mathematics, vol. 13, Springer-Verlag, Berlin, 1990, An introduction.

[3] Marek Byrtus and Bohumír Bastl, $G^{1}$ Hermite interpolation by PH cubics revisited, Comput. Aided Geom. Design 27 (2010), no. 8, 622-630.

[4] Hyeong In Choi, Rida T. Farouki, Song-Hwa Kwon, and Hwan Pyo Moon, Topological criterion for selection of quintic Pythagorean-hodograph Hermite interpolants, Comput. Aided Geom. Design 25 (2008), no. 6, 411-433.

[5] Hyeong In Choi, Doo Seok Lee, and Hwan Pyo Moon, Clifford algebra, spin representation, and rational parameterization of curves and surfaces, Adv. Comput. Math. 17 (2002), no. 12, 5-48, Advances in geometrical algorithms and representations.

[6] Gerald Farin, Josef Hoschek, and Myung-Soo Kim, Handbook of Computer Aided Geometric Design, first ed., Elsevier, Amsterdam, 2002.

[7] Rida T. Farouki, The conformal map $z \rightarrow z^{2}$ of the hodograph plane, Comput. Aided Geom. Design 11 (1994), no. 4, 363-390.

[8] __ Pythagorean-hodograph curves: algebra and geometry inseparable, Geometry and Computing, vol. 1, Springer, Berlin, 2008.

[9] Rida T. Farouki, Carlotta Giannelli, Carla Manni, and Alessandra Sestini, Identification of spatial PH quintic Hermite interpolants with near-optimal shape measures, Comput. Aided Geom. Design 25 (2008), no. 4-5, 274-297.

[10] Rida T. Farouki and Takis Sakkalis, Pythagorean hodographs, IBM J. Res. Develop. 34 (1990), no. 5, 736-752.

[11] Chang Yong Han, Geometric Hermite interpolation by monotone helical quintics, Comput. Aided Geom. Design 27 (2010), no. 9, 713-719.

[12] Gašper Jaklič, Jernej Kozak, Marjeta Krajnc, Vito Vitrih, and Emil Žagar, Geometric Lagrange interpolation by planar cubic Pythagorean-hodograph curves, Comput. Aided Geom. Design 25 (2008), no. 9, 720-728.

[13] On interpolation by planar cubic $G^{2}$ Pythagorean-hodograph spline curves, Math. Comp. 79 (2010), no. 269, 305-326.

[14] — An approach to geometric interpolation by Pythagorean-hodograph curves, Adv. Comput. Math. (2012), no. 37, 123-150.

[15] B. Jüttler, Hermite interpolation by Pythagorean hodograph curves of degree seven, Math. Comp. 70 (2001), no. 235, 1089-1111 (electronic).

[16] Bert Jüttler and C Mäurer, Cubic Pythagorean-hodograph spline curves and applications to sweep surface modeling, Comput. Aided Geom. Design 31 (1999), 73-83.

[17] Song-Hwa Kwon, Solvability of $G^{1}$ Hermite interpolation by spatial Pythagorean-hodograph cubics and its selection scheme, Comput. Aided Geom. Design 27 (2010), no. 2, 138-149.

[18] Tom Lyche and Knut Mørken, A metric for parametric approximation, Curves and surfaces in geometric design (Chamonix-Mont-Blanc, 1993), A K Peters, Wellesley, MA, 1994, pp. 311-318.

[19] Dereck S. Meek and D. J. Walton, Hermite interpolation with Tschirnhausen cubic spirals, Comput. Aided Geom. Design 14 (1997), no. 7, 619-635.

[20] Dereck S. Meek and D. J. Walton, $G^{2}$ curve design with a pair of Pythagorean hodograph quintic spiral segments, Comput. Aided Geom. Design 24 (2007), no. 5, 267-285.

[21] Francesca Pelosi, Rida T. Farouki, Carla Manni, and Alessandra Sestini, Geometric Hermite 
interpolation by spatial Pythagorean-hodograph cubics, Adv. Comput. Math. 22 (2005), no. 4, 325-352.

[22] Takis Sakkalis and Rida T. Farouki, Pythagorean-hodograph curves in Euclidean spaces of dimension greater than 3, J. Comput. Appl. Math. 236 (2012), no. 17, 4375-4382.

[23] Zbyněk Šír and Bert Jüttler, $C^{2}$ Hermite interpolation by Pythagorean hodograph space curves, Math. Comp. 76 (2007), no. 259, 1373-1391. 